(Aus der physiologischen Abteilung der zoologischen Station zu Neapel und der zoologischen Station zu Triest.)

\title{
Gibt es in der Muskulatur der Mollusken. periphere, kontinuierlich leitende Nervennetze bei Abwesenheit von Ganglienzellen?
}

\section{Untersnchungen an Cephalopoden. Von}

F. B. Hofmann (Innsbruck).

(Mit 1 Textfigur und Tafel VI.):

In einer Abhandlung über das intrakardiale Nervensystem des: Frosches ${ }^{1}$ ) hatte ich darauf hingewiesen, dass das reiche Nervengeflecht, welches die Herzmuskulatur allenthalben durchzieht, an Stellen, wo es durch die Golgi-Methode besonders gut dargestellt ist, keine freien, sogenannten knöpfchenförmigen Endigungen aufwies, sondern dass sich die intramuskulären Nervenfädchen ganz kontinuierlich von Muskelzelle zu Muskelzelle weiter verfolgen liessen, sowie dies schon Ranvier an Goldpräparaten beschrieben hatte. Ich hatte daraus den Schluss gezogen, dass innerhalb des Herzmuskels ein wahres Endnetz mindestens zwischen den Teilästen jeder einzelnen Nervenfaser unter sich bestehen müsse. Dagegen. blieb unentschieden, ob bloss eine Schlingenbildung zwischen den Endästchen jeder Nervenfaser unter sich, oder aber ein durchgehendes, kontinuierliches Nervennetz, gebildet durch Anastomosen aller zur Muskulatur hinziehenden Nervenfasern untereinander vorbanden sei. Später hat dann $\mathrm{B} \mathrm{eth}^{2}$ ) angegeben, dass im Herzen (des Frosehes) ein kontinuierliches Nervennetz vorhanden sei. Aber das von Bethe. angegebene Nervennetz ist etwas prinzipiell ganz anderes als das

1) His' Arch. f. Anat: 1902. S. 54.

2) Allgemeine Anatomie und Physiologie des Nervensystems. Thième, Leipzig 1908. 
von mir als möglich erschlossene. Das Bethe'sche Nervennetz soll aus anastomosierenden Ganglienzellen bestehen, die sich im sogenannten Grundplexus und perimuskulären Plexus befinden, während das von mir als möglich angenommene Nervennetz von Ganglienzellen ganz unabhängig und streng intramuskulär wäre ${ }^{1}$ ). $\mathrm{Ob}$ es zugleich auch schon die allerletzte Endigung der Nerven an der Muskulatur darstellt, oder ob von seinen Fädchen aus noch kurze, frei endigende Seitenzweigchen in die Muskelfasern selbst hineingehen, so dass es also dann eigentlich als ein präterminales Netz zu bezeichnen wäre, lasse ich vorläufig noch offen. Freilich habe ich von solchen Seitenästchen bisher weder mit der Golgi-noch mit der Methylenblaumethode etwas gesehen ${ }^{2}$ ), ebensowenig als sie Ranvier mit der Goldmethode darstellen konnte.

Ganz analoge Verhältnisse, wie am Herzmuskel der Amphibien habe ich nun auch an der Chromatophoren-, Haut-, Flossen- und Mantelmuskulatur der Cephalopoden vorgefunden und berichte darüber in einer gleichzeitig im Arch. f. mikrosk. Anatomie erscheinenden Abhandlung. Insbesondere gelang mir an den Chromatophoren der Nachweis, dass die Nervenfasern nicht an ihnen endigen, sondern, nachdem sie an den Pigmentkörper und die Zellkerne nahe herangetreten sind, wieder schleifenförmig umbiegen und von ihnen wegziehen. Es erhob sich also auch für die Innervation der Chromatophorenmuskulatur dieselbe Frage wie für die Herzmuskulatur der Amphibien. Ganz Analoges gilt, wie in der erwähnten Abhandlung ausgeführt wird, sehr wahrseheinlich auch für die Flossen- und Mantelmuskulatur der Cephalopoden, sowie für die glatte Muskulatur der Wirbeltiere. Überall muss man dann die gleiche Alternativfrage aufwerfen: Bilden die Endverzweigungen einer jeden Stammnervenfaser ${ }^{3}$ ) bloss unter sich geschlossene Schleifen, oder besteht ein durchgehendes, kontinuierliches, intramuskuläres Nervennetz, enstanden durch Anastomosen der Teiläste aller Nervenfasern untereinander? Rein histologisch liess sich diese Frage an

1) Genaueres darüber veröffentliche ich gleichzeitig im Arch. f. mikrosk. Anat. Ich bin der Meinung, dass Bethe's Annahme spezifischer gangliöser Nervennetze in der Herz- und Gefässmuskulatur der Wirbeltiere auf irrtümlicher Deutung der Methylenblaubilder beruht.

2) Vgl, meine frühere Abhandlung $1902 \mathrm{~S}, 103 \mathrm{ff}$.

3) Mit diesem Worte bezeichne ich die aus dem Nervenfortsatz einer Ganglienzelle entstehende noch ungeteilte Nervenfaser. 
Gibt es in der Muskulatur der Mollusken periphere Nervennetze etc.? 377

meinen Objekten nicht entscheiden. Höchstens konnte man aus Analogiegründen, insbesondere nach Analogie mit den Verhältnissen im Zentralnervensystem ${ }^{1}$ ), den zweiten Teil der Alternative für wahrscheinlicher halten. Aber es bestand die Möglichkeit, dass vielleicht physiologische Experimente eine Entscheidung bringen konnten. Es war ja denkbar, dass im kontinuierlichen Nervennetz - falls es besteht - streng lokal an einer Stelle gesetzte Erregungen sich nach allen Richtungen bin ausbreiten, dass also dem kontinuierlichen anatomischen Netz, physiologisch eine allseitige Fortleitung der Erregung entspreche. Eine solche ist zwar in der glatten Muskulatur der Wirbeltiere im allgemeinen nicht vorhanden, aber in der Muskulatur gewisser wirbelloser Tiere wurde sie von Bethe (1. c.) tatsächlich angegeben und auf die Anwesenheit seiner, allerdings ganz anders gearteten Nervennetze bezogen. Ich benutzte daher einen Aufenthalt an der zoologischen Station in Neapel zu Ostern 1904, der mir durch ein Stipendium aus den Mitteln der Leipziger AlbrechtStiftung ermöglicht wurde, um zugleich mit den schon erwäbnten bistologischen Untersuchungen auch physiologische Beobachtungen an denselben Objekten anzustellen und so durch den Vergleich beider womöglich Klarheit zu erlangen. Diese physiologischen Experimente habe ich zu Ostern 1906 an der zoologischen Station in Triest nochmals nachgeprüft und erweitert, and ich ergreife gerne die Gelegenheit, den Herren Dr. Lo Bianco, Dr. Burian und Dr. Bauer von der zoologischen Station zu Neapel sowie Herrn Professor Cori, dem Leiter der zoologischen Station zu Triest, für ihre bereitwillige Unterstützung meinen besten Dank zu sagen.

\section{Die Mantelmuskulatur von Eledone.}

Dass sich in der Mantelmuskulatur der Cephalopoden - als spezielles Untersuchungsobjekt diente Eledone moschata ${ }^{2}$ ) - ein kontinuierliches Nervennetz befindet, durch welches die Erregung von jeder Stelle aus ebenso rasch und gut über den ganzen Mantel hin fortgeleitet würde wie in den Nervenstämmen, das war von vorne herein eine unmögliche Annahme. Das würde nämlich heissen,

1) Darauf sowie auf die Beziehungen zur Apáthy'schen Neurofibrillenlehre komme ich in einer späteren Abhandlung zu sprechen.

2) Zur ersten Orientierung über die hier vorliegenden anatomisch-physio-

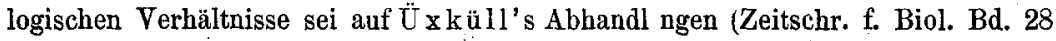
S. 550 und Bd. 30 S. 317) verwiesen. 
dass die Tiere die Mantelmuskulatur bei anhaltender Innervation bloss in einen einzigen gleichmässigen Kontraktionszustand versetzen könnten, denn wo immer die natürliche Erregung am Mantel angreifen würde, stets würde sie sich nach dieser Aunahme über die gesamte vorhandene Muskulatur hin ausbreiten. Nun vermögen aber die Tiere die Mantelmuskulatur in sehr verschiedener Weise dauernd kontrahiert $\mathrm{zu}$ halten. So z. B. halten sie nach dem Herausnehmen aus dem Wasser öfter den Mantel längere Zeit ganz weit offen, um ihn dann mit einem Ruck plötzlich zusammenfallen zu lassen ${ }^{1}$ ); andererseits aber können sie ebenso dauernd den vorderen Mantelrand um den Eingeweidesack geschlossen halten (als Reflex auf sensible Reizung der Innenseite der Mantelöffnung) usf. Demnach wäre schon nach der blossen Beobachtung der spontanen Bewegungen am lebenden Tiere die Annahme einer diffusen Erregungsleitung innerhalb der Mantelmuskulatur bloss unter einer bestimmten Voraussetzung haltbar. Bezeichnen wir die Stelle, an welcher die Erregung in das hypothetische erregungsleitende Nervennetz der Muskulatur eintritt, sei es infolge Erregung eines Nervenstammes, sei es infolge direkter Reizung der Muskulatur, als die Einbruchsstelle der Erregung ins Nervennetz, so kann man diese Voraussetzung kurz so formulieren, dass sich die Erregung im Nervennetz von der Einbruchsstelle aus mit sehr grossem Dekrement fortpflanzen müsste, d. h. also, dass sie an der Einbruchsstelle am stärksten wäre, und mit der Entfernung von ihr sehr rasch an Stärke abnehme. Bei der ausserordentlich dichten Struktur und der innigen Verfilzung der Muskelbündel des Mantels ist es nun so gut wie unmöglich, zu ent-

1) Es ist dies nichts anderes als die Fluchtbewegung der Tiere. Dabei öffnen sie im Wasser zunächst weit die Mantelhöhle, füllen sie so mit Wasser, stossen dieses, indem sie den Mantel vorn abschliessen, durch den Trichter heraus und treiben sich durch den Rückstoss rasch fort (unter Verlängerung der Mantelachse vgl. v. Üxküll, Zeitschr. f. Biol. Bd. 28 S. 563 und die genaue Analyse der Schwimm- und Atembewegungen der Eledone von demselben, ebenda Bd. 31 S. $591 \mathrm{ff}$. 1894). Die Öffnung des Mantels scheint (besonders bei Sepia) an der Luft viel krampfhafter zu sein und länger anzuhalten als im Wasser, vielleicht deshalb, weil durch das eindringende Seewasser sensible Nerven gereizt werden, durch deren Erregung die Füllung des Mantelsacks angezeigt wird. Fällt diese Erregung fort, so dehnt sich anscheinend die Einsaugstellung der Mantelmuskulatur so lange aus, wie die Inspirationsbewegung beim Säugetier etwa bei doppelseitigem Pneumothorax. Wenn der Zusammenhang so richtig gedeutet ist, so wäre das ein interessantes Analogon zur Selbststeuerung der Atmung von Hering. 
scheiden, ob sich die Erregung in der Mantelmuskulatur von der Einbruchsstelle aus noch eine kleine Strecke weiter fortpflanzt oder nicht, wenigstens wenn man den mechanischen Kontraktionsakt als Kriterium der Erregung benutzt. Durch die Kontraktion einer Stelle des aufgeschnittenen und ausgebreiteten Mantels wird nämlich die Umgebung so stark verschoben, dass man gar nicht imstande ist, durch blosse Beobachtung der Bewegung - und ebensowenig durch graphische Verzeichnung (ich habe mich erfolglos bemüht, dies durch Registrierung von Verdickungskurven $\mathrm{zu}$ erzielen) - die Grenzen zwischen der aktiv kontrahierten Stelle und den passiv mitgezogenen Teilen auch nur einigermaassen scharf $\mathrm{zu}$ bestimmen. Man kann auf diese Weise, besonders wenn man den aufgeklappten Mantel in radiärer Kichtung vom Rande her gegen das Stellarganglion etwas einkerbt, bloss feststellen, dass durch Reizung der einzelnen vom Stellarganglion abgehenden Nervenbündel eine stärkste Kontraktion an der Eintrittsstelle des Nerven in die Muskulatur erfolgt, in der Umgebung dagegen eine mit der Entfernung abnehmend schwächere Bewegung zu sehen ist. Das ergibt uns aber nicht mehr als die einleitenden Überlegungen nach den Beobachtungen am lebenden Tiere und kann allenfalls, wenn man dies für notwendig halten sollte, als eine experimentelle Bestätigung derselben aufgefasst werden.

Eher wäre es vielleicht möglich, mit Hilfe der Ableitung von Aktionsströmen die Grenzen des wirklichen Erregungsgebietes zu bestimmen. Aber das wäre eine sehr umständliche und zeitraubende Arbeit gewesen, die ausserdem dadurch überflüssig wurde, dass sich an anderen Objekten die Abgrenzung viel leichter und sicherer feststellen liess.

\section{Die Flossenmuskulatur von Sepia.}

Als weitaus geeigneter für das Studium der Frage nach der Möglichkeit einer peripheren Erregungsleitung im Nervennetz musste die Muskulatur der Flosse erscheinen, welche bei Sepia den ganzen Rand des Mantels vom oralen bis zum apikalen Pole umsäumt. Die Bewegungen dieser Flosse beim Schwimmen stellen sich dar als abwechselnde Senkungen und Hebungen, welche in Form schön geschwungener Wellen entweder vom oralen zum apikalen Ende oder aber in entgegengesetzter Richtung ablaufen ${ }^{1}$ ). Augenblicksbilder

1) Vgl. Brehm's Tierleben, 2. Aufl., Bd. 10 S. 207. 1878. 
solcher Wellen sind in den Aufnahmen (Taf. VI) der folgenden Abhandlung abgebildet. Man sieht hier, besonders schön in Fig. 1 u. 2, dass sich zwei Wellentäler aufeinanderfolgen können, und man kann weiter konstatieren, dass die Bewegung entweder in einer kräftigen Umbiegung der Flosse nach unten besteht, mit nachheriger Erhebung zur Horizontale (Fig. 1) oder auch in einem Schlag von der Horizontale nach oben. Durch die erstere Bewegungsform steigen die Tiere im Wasser auf, durch die letztere senken sie sich zu Boden.

Was die Anordnung der Muskulatur in der Flosse betrifft, so können wị, wie ich schon im Arch. f. mikr. Anat. Bd. 70 kurz erwähnt habe, als hauptsächlichste Schichten zunächst solche unterscheiden, welche vom Ansatz der Flosse am Mantel radiär zum freien Rande hinziehen; wir wollen sie als Radiärmuskeln bezeichnen. Diese Züge wechseln in regelmässiger Weise ab mit Muskelschichten, deren Elemente senkrecht zur Oberfläche der Flosse in die Tiefe hineinziehen, „transversale". Muskelschichten (man vgl. dazu Fig. 7 meiner Abhandlung im Arch. f. mikr. Anat.). Daneben kommen im Inneren der Flosse auch noch longitudinal von vorne nach hinten verlaufende Muskelfaserzüge vor. Ganz absehen will ich von den oberflächlichen, der Subkutis angehörenden Muskelzügen. Die radiären Muskelschichten anaștomosieren, wie man schon in der Aufsicht auf die Flossenmuskulatur sehen kann, sehr häufig miteinander durch schräg verlaufende Muskelzüge. Gewiss befinden sich solche Verbindungen, welche die trennenden transversalen Muskelsehichten durchsetzen, auch noch in der Tiefe, doch kann ich dies nicht mit Sicherheit nachweisen. Diese Anastomosen sind deswegen wichtig, weil das von mir als möglich erschlossene periphere Nervennetz ja 'stets nur zusammen mit den Muskélfasern verläuft. Wir können daher nach meiner Auffassung eine Erregungsleitung in diesem Nervennetz auch nur da erwarten, wo Anastomosen der Muskelzüge vorhanden sind. Gerade hierin liegt ja der prinzipielle Unterschied meiner Auffassung gegenüber der von Bethe.

Will man sich aus der Anordnung der Muskulatur eine Vorstellung rom Zustandekommen des schönen Wellenschlages der Flosse bilden, so muss man nach dem Gesagten wohl annehmen, dass der Schlag nach unten durch eine Kontraktion der Radiärmuskelzüge auf der Unterseite der Flosse entsteht, wobei die Radiärmuskeln der Oberseite erschlafft sind, Ein Schlagen nach oben würde bewirkt 
werden durch Kontraktion der Radiärmuskeln der oberen Seite unter Erschlaffung der Radiärmuskeln der Unterseite. Mitbeteiligt am Sehwimmschlag sind wohl auch die Tranversalmuskeln, deren Kontraktion die Flossendicke vermindert und die Flosse dementsprechend verbreitert und wohl auch versteift. Ob die inneren Longitudinalmuskeln dabei, irgendeine Rolle spielen, ist schwer zu sagen.

Es fragte sich nun, ob sich die oben zunächst beschriebene wellenförmig "wandernde Flossenbewegung durch einen einfachen Leitungsvorgang in einem kontinuierlichen peripheren Nervennetz erklären liesse. Die Frage schien um so berechtigter, als ja Bethe (l. c. S. 113 ff.) eine sehr ähnliche Bewegungsform, den Schlag der Mantellappen von Aplysia, auf Fortleitung der Erregung in einem peripheren Nervennetz zurückführt. Ich werde mich mit diesem Objekt in einer späteren Abhandlung eingehender beschäftigen und nehme hier gleich den Hauptgrund vorweg, aus welchem sich die Flossenbewegung bei Sepia für das Studium dieser Frage besser eignet als die Mantellappen von Aplysia: das ist das Fehlen eines peripheren Reflexapparates bei. unserem Objekt.

Es lässt sich nun leicht nachweisen, dass das Wandern der Kontraktionswelle in der Flosse von Sepia nicht auf Erregungsleitung in der Peripherie beruht, oder mit anderen Worten, dass die Koordination der Muskelkontraktionen beim. Flossenschlage nicht auf einer peripheren, sondern auf einer zentralen Organisation beruht. Der Beweis lässt sich auf doppelte Weise erbringen: einmal durch Reizung: der einzelnen Nervenäste, die zur Flossenmuskulatur hinziehen, zweitens durch partielle Lähmung der Flossenmuskulatur.

Bei Reizung der einzelnen Flossennerven, welche mit den Chromatophoren- und Hautmuskelnerven vermischt nach Durch, bohrung des Mantels radienförmig gegen die Flosse zu ausstrahlen (das Genauere darüber folgt später im dritten Abschnitte), kann man ausnahmslos beobachten, dass auf Reizung eines bestimmten Nerven hin: immer auch eine ganz bestimmte Partie der Flosse sich bewegt, und dass sich. die Erregung von hier aus niemals auf die übrigen Teile der Flosse ausbreitet. Die Bewegungen, die man erhält, sind übrigens, wie nicht anders zu erwarten ist, keine besonders charakteristischen. Das rührt zum Teil davon her, dass ich die Untersuchung so vornahm, dass die Flosse flach auf einer ebenen Unterlage (Glasplatte) auflag. Man kann dann natürich nur ein Heben derselben oder ein Plattwerden und Sichausbreiten wahrnehmen. Es ist aber 
wahrscheinlich, dass man auch dann, wenn man die Experimente mit im Wasser freischwebender Flosse vornehmen würde, keine so wohl koordinierte Bewegung, wie es die Schwimmbewegung ist, durch Reizung eines peripheren Nerven auslösen könnte. Ich komme darauf in einer folgenden Abhandlung über die Schwimmbewegung bei Aplysia noch zurück. Selbstverständlich ist es auch, dass man an der Flosse die Grenzen der sich kontrahierenden Muskelpartie nicht ganz genau angeben kann, weil, wie beim Mantel, die Umgebung eine Strecke weit mit bewegt wird. Im ganzen kommt man also durch die Reizungsversuche an der Flosse zu demselben Schluss wie am Mantel: Hier wie dort lässt sich die Annahme einer peripheren kontinuierlichen Erregungsleitung nur unter der Bedingung halten, dass sich die Erregung mit sehr starkem Dekrement fortpflanzt und demgemäss in sehr kurzer Entfernung von der Einbruchsstelle in das hypothetische Nervennetz schon erlischt.

Der noch mögliche Einwand, dass die Reizversuche an toten Tieren ausgeführt wurden, bei welchen die Reizbarkeit und Leitfähigkeit der Nervennetze schon gelitten haben kann, bei welchen also das Dekrement erst nach dem Tode aufgetreten sein könnte, wird erledigt durch die Beobachtungen an lebenden Tieren mit partieller Flossenlähmung. Es gelang mir nach eiuiger Übung, einzelnen Tieren bloss die Nerven für die vordersten oder die für die hintersten Partien der Flosse zu durchschneiden. (Bloss die Nerven für die mittleren Partien unter Erhaltung der vorderen und hinteren auszuschalten, ist mir bisher nicht gelungen, aber nur deswegen, weil man bei der Operation ziemlich im Blinden arbeitet und es daher stark vom Zufall abhängt, welche Nerven man trifft.) Gleich nach der Operation kann man nun auf das schönste beobachten, dass sich das graziöse Wellenspiel der normalen Flossenteile auf die gelähmte Partie nicht fortsetzt. Nur jene Stellen der gelähmten Flossenpartie, welche an die normale angrenzen, werden durch die Schwingungen des normalen Teiles rein passiv etwas mitgerissen, was man aber vom aktiven Spiel wohl unterscheiden kann.

In der folgenden Abhandlung wird gezeigt werden, dass sich im peripheren motorischen Apparate der Chromatophoren nach der Nervendurchschneidung allmählich eine Steigerung der mechanisehen Reizbarkeit ausbildet, welche wahrscheinlich die Nervenfasern betrifft, und mit der zu gleicher Zeit eine tonische Kontraktion der Chromatophoreumuskeln einhergeht. Diese Dauerkontraktion setzt nach der 
Lähmung wohl auch in der Flossenmuskulatur ein, wenigstens ist die gelähmte Flosse regelmässig viel schmäler als die der normalén Seite (vgl. das hintere Ende von Fig. 3 auf Taf. VI). Aber selbst dann, wenn auf diese Weise anscheinend ungemein günstige Bedingungen für die Leitung der Erregung im supponierten Nervennetz gegeben sind, selbst dann ist von einem wellenförmigen Fortschreiten der Erregung in das gelähmte Gebiet hinein keine Rede. Es wird in dieser folgenden Abhandlung auch gezeigt werden, warum es nicht angeht, den dauernden peripheren Tonus der gelähmten Muskulatur etwa auf ständig von den normalen Partien ausgehende Erregungswellen zurückzuführen, und es lässt sich daher das bisher Bekannte allgemein dahin zusammenfassen, dass man in der Flossenmuskulatur von Sepia nie eine periphere Fortpflanzung der an einer Stelle gesetzten Erregung über die ganze Flosse hin beobachten kann. Das normale Wellenspiel der Flosse kann daher, wie oben schon gesagt wurde, nicht auf einer peripheren Erregungsleitung in einem Nervennetz beruhen, sondern muss in der Organisation des Zentralnervensystems begründet sein. Sollte es nun innerhalb der Flossenmuskulatur trotzdem ein kontinuierlich zusammenhängendes Nervennetz geben, so würde aus den mitgeteilten Tatsachen folgen, dass sich die Erregung in ihm höchstens mit einem starken Dekrement fortpflanzen könnte. Diese Folgerung ergab sich auch schon aus den Versuchen an der Mantelmuskulatur und kann an der Flosse nur viel besser bewiesen werden.

Ein Umstand allerdings beeinträchtigt die Stichhaltigkeit der eben gezogenen Folgerung sehr: Es fragt sich, ob denn auch wirklich die Muskelschichten der Flosșe so ausgedehnt miteinander anastomosieren, dass eine allseitige Verknüpfung der Nervennetze der verschiedenen Muskelschichten anzunehmen ist. Bei den Radiärmuskelschichten begegnet man in der Aufsicht sehr häufig einer völligen Trennung durch Zwischenschaltung von Transversalmuskeln. Dass hier eine Anastomosierung in der Tiefe stattfindet, ist zwar wahrscheinlich, aber noch nicht sicher bewiesen. Ich möchte hinzufügen, dass diese Überlegung auch für die ähnlich angeordnete Muskulatur des Mantels gilt. 


\section{Die Chromatophorenmuskulatur von Sepia (nebst Bemerkungen iiber den Hantmuskel).}

\section{A. Die Verhältnisse am Chromatophorenapparate unter normalen Bedingungen.}

Weitaus das bequemste und günstigste Objekt für das Studium der Frage nach der Ausbreitung der Erregung in einem peripheren Nervennetz bildet die Chromatophorenmuskulatur der Cephalopoden. Am geeignetsten für meine Untersuchungen hat sich von den verschiedenen Arten Sepia officinalis erwiesen, weil es hier verhältnismässig leicht gelingt, die Chromatophorennerven $\mathrm{zu}$ isolieren und elektrisch zu reizen, und weil jhre Reizbarkeit auch nach der Präparation, wenn man die Präparate in Seewasser aufbewahrt, noch stundenlang ziemlich unverändert bestehen bleibt. Man kann hier ungefähr ebenso bequem arbeiten wie am Nervmuskelpräparate des Frosches, ja vielleicht noch bequemer insofern, als man das Tier nach dem Tode, wenn man es in durchlüftetem Seewasser schwimmen lässt, ganz' gut noch nach einigen Stunden in Arbeit nehmen kann. Verhältnismässig leicht ist die Präparation der Nerven auch bei Loligo vulgaris. Nur sind die Nerven hier viel zarter und werden beim Präparieren leicht geschädigt, ferner ist das Arbeiten bei dieser Art insofern unbequemer, als die Tiere sehr rasch absterben und der Chromatophorenapparat nach dem Tode bedeutend schneller seine Rejzbarkeit einbüsst als bei Sepia.

Auf die Histologie der Chromatophoren gehe ich bier nicht weiter ein, da ich die histologischen Vorfragen für diese Untersuchung schon eingehend im Arch. f. mikr. Anat. Bd. 70 erörtere. Die Literatur über den Gegenständ findet der Leser vollständig bei G. v. Rynberk in den Ergebnissen d. Physiol. Bd. 5 S. $353 \mathrm{ff}$. 1906. Hier soll nur, als auf den wichtigsten Punkt für das Verständnis des Folgenden, darauf hingewiesen werden, dass die Expansion der Chromatophoren bekanntlich durch Kontraktion der am Pigmentkörper der Chromatophoren inserierenden Radiärmuskeln herbeigeführt wird, durch deren Zug die vorher zu einem rnakroskopisch eben sichtbaren dunklen Pünktchen zusammengeballten Pigmentkörper zu flachen Scheibchen ausgezogen werden; deren Ränder sich beî maximaler Expansion überdecken, so dass die Haut ganz dunkel wird.: Bei Erschlaffung der Radiärmuskeln erfolgt wiederum Zusammenballung des Pigments, also Bleichung der Haut. Welche Kräfte dabei wirksam sind, darüber siehe v. Rynberk und meine folgende Abhandlung. Was die Farbe der Chromatophoren betrifft, so finden sich bei Loligo vulgaris Chromatophoren dreierlei Art: solche, welche im expandierten Zustande hellgelb, solche, welche hellviolett und solche, welche rostbraun aussehen. Im zusammengeballten Zustande sehen die beiden 
letzten Arten unter dem Mikroskop gleich schwarz aus. Béi Sepia officinalis finde ich bloss hellgelbe und ganz dunkle, schwach violette Chromatophoren. Beide Arten liegen in verschiedener Höhe, eine über der anderen. Dass B oll (Beiträge zur vergl. Histologie des Molluskentypus. Arch. f. mikr. Anat. Bd. 4 Suppl. S. 64. 1869) sowohl bei Sepia als bei Loligo die hellgelben Chromatophoren übersehen hat, ist mir ganz unerklärlich. Bei gleichzeitiger Expansion der gelben und der dunkelvioletten Chromatophoren von Sepia werden die gelben von den violetten so unterdrückt, dass man ihr Verhalten kaum studieren kann. Wenn aber der Farbenton der Haut bei der Dunkelung ein rein brauner ist, ohne Stich ins Violette, so ist wohl anzunehmen, dass dieser Ton durch die gleichzeitige Expansion der gelben und violetten Chromatophoren erzengt wird. Über isolierte Kontraktion der gelben Chromatophoren bei Sepia officinalis siehe weiter unten S. 403.

Für das Studium der uns hier interessierenden Frage bieten nun die Chromatophorenmuskeln den Vorteil, dass man bei Sepia und Loligo ganz gut schon mit freiem Auge, noch besser aber unter Zuhilfenahme einer Lupe, das Verhalten der einzelnen Chromatophoren, zum mindesten der dunkel violetten feststellen kann. Nun besteht der Muskelapparat einer Chromatophore aus einer Gruppe von durchschnittlich etwa 20 Radiärfasern, welche nach den Untersuchungen von $\mathrm{Chun}^{\mathbf{1}}$ ) als Ausläufer einer einzigen vielkernigen Zelle aufzufassen sind. Aber selbst wenn man nach anderen Autoren jeder einzelnen Radiärfaser den Charakter einer Zelle zuerkennen wollte, so würde die breite Verbindungsbrücke zwischen den einzelnen Radiärmuskelfasern dazu zwingen, die Chromatophoren mindestens als ein einheitlich funktionierendes Syncytium mehrerer miteinander verbundener Zellen aufzufassen. Wir haben also bei der Beobachtung der Chromatophoren den Vorteil, dass wir die Grenze der Ausbreitung der Erregung mit grosser Schärfe bis zu den funktionellen Einheiten hin bestimmen können. Man kann ferner schon durch die blosse Beobachtung ein eventuelles Fortschreiten der Erregung von Chromatophore zu Chromatophore feststellen. Alle diese Beobachtungen würden endlich auch ziemlich leicht photographisch festzuhalten sein, ja man könnte gewiss von fortschreitenden Bewegungen ohne allzu grosse Schwierigkeit auch kinematographische oder ähnliche Aufnahmen herstellen. Bei dem ersten Versuche zur Orientierung verbot sich schon wegen Zeitmangels die Anwendung aller dieser etwas umständlichen Manipulationen.

1) Über die Chromatophoren der Cephalopoden. Verhandl. d. Deutschen zoolog. Gesellsch. 12. Jahresvers. S. 162. 1902. 
Ich habe mich vielmehr auf die blosse Beobachtung mit freiem Auge oder mit der Lupe beschränkt und meine Beobachtungen noch während des Versuchs oder unmittelbar danach protokolliert und eventuell rasch skizziert. Im folgenden werde ich mich bei der Beschreibung der Tatsachen, um ihre treue Wiedergabe nicht etwa durch subjektive spätere Überlegungen zu fälschen, möglichst dem Wortlaute nach an diese Protokolle halten.

Die Präparation der Chromatophorennerven bei Sepia officinalis habe ich in der Regel so vorgenommen, dass ich vom vorderen Rande des Mantels her dicht neben dem Rückenschulp mit der Schere einen Einschnitt nach hinten zu bis etwas hinter das Stellarganglion machte. Dann wurde der Mantelnerv vor dem Stellarganglion mit einem Faden unterbunden und, wäbrend er am Faden gehalten wurde, mit der Schere auf seinem Wege durch das Loch, in welchem er die Mantelmuskulatur (zusammen mit den Blutgefässen) durchsetzt, frei präpariert. Unmittelbar nach dem Durchtritt durch die derbe Mantelmuskulatur teilt sich nun der Nerv in eine grosse Zahl radiär auseinanderschiessender dicker und dünner Äste, welche untereinander durch Bindegewebe zusammengehalten werden und auf die mannigfaltigste Weise miteinander anastomosieren, so dass aus Bindegewebe und Nervenbündeln eine förmliche Schicht entsteht, welche der Mantelmuskulatur nach aussen zu unmittelbar aufliegt, aber sehr leicht von ihr abpräpariert werden kann. Ziebt man nunmehr die nach aussen zu auf die Nerven aufgelagerte Hautmuskelschicht $a b$, so sieht man jetzt die einzelnen Nervenäste nur noch durch wenig Bindegewebe miteinander verbunden und kann sie leicht mit Pinzette und Schere auf grössere Strecken hin in getrennte Nerven zerteilen, wobei freilich viele feine Anastomosen durchtrennt werden. Ich pflegte sodanı die einzelnen dickeren Nervenbündel von neuem isoliert zu unterbinden und sie in der Richtung von vorn nach hinten mit einzelnen Nummern zu bezeichnen, als Nerv 1 für die vorderste Partie der Flosse und Haut, Nerv 2 für die nächstanschliessende Partie usw., wobei aber zu berücksichtigen ist, dass infolge der mannigfachen Anastomosierung und Aufsplitterung der Nerven in der Regel einige zwischenliegende feinere Ästchen bei der Präparation zerstört werden.

Will man sich nunmehr einen Überblick über die von den einzelnen isolierten Nervenbündeln versorgten Chromatophoren-(Haut-) Bezirke verschaffen, so ist es zweckmässig, die frei präparierten 
Nerven möglichst peripher mit starken Strömen zu reizen, weil in der Nähe der Unterbindungsstelle sich die unvermeidlichen Schädigungen bei der Präparation (besonders gegen Zerrung sind die Nerven sehr empfindlich!) störend geltend machen. Ich benutzte zur Reizung einen grossen Schlittenapparat mit zwei Daniell'schen Elementen im primären Kreis. Die Reizschwelle lag bei meiner Anordnung in der Regel zwischen 20 und $35 \mathrm{~cm}$ Abstand der sekundären Spirale (Rollenabstand $=$ R.-A.), bei besonders gut reizbaren Nerven auch noch niedriger.

Da die verschiedenen Nervenbündel in ihrer Dicke sehr beträchtlich variierten, so variierte die Schwelle wegen der versehiedenen Stromdichte natürlich auch mit der Dicke des Bündels. Die dünnsten Bündel gerieten mitunter schon um $40 \mathrm{~cm}$ R.-A. herum in maximale Erregung.

Die Vorgänge bei schwachen, eben über der Schwelle liegenden Reizungen sollen erst später besprochen werden. Wir halten uns zunächst an die Beobachtungen bei maximaler Reizung. Diese ergeben nun als erstes Hauptresultat, dass jeder einzelne maximal gereizte Nerv nur bestimmte und bei wiederholter Reizung stets gleiche Chromatophorengruppen zur Expansion bringt, während die Chromatophoren der Umgebung in gar keiner Weise beeinflusst werden. Die von einem Nerven aus beeinflussbaren Chromatophoren liegen gewöhnlich in bestimmten Hautbezirken dicht nebeneinander, doch erstreckt sich der Nerveneinfluss in der Regel nicht auf alle Chromatophoren der betreffenden Hautfläche; es bleiben darin "Inseln" nicht expandierter Chromatophoren übrig (s. darüber unten S. 389). Wenn wir daher unser Augenmerk auf die innervierten Hautbezirke richten, so müssen wir sagen, dass jedem Nerv zwar ein bestimmter Innervationsbezirk von konstanter Begrenzung auf der Haut entspricht, der aber gewisse, auch konstante, ausgesparte Lücken enthält. Die Konstanz der Innervationsbezirke bei wiederholter Reizung habe ich freilich nur durch den blossen Augenschein festgestellt, wobei als Anbaltspunkte für die Grenze insbesondere jene weissen Streifen und Flecken auf der Haut von Sepia dienten, welche durch besonders reichliche Einlagerung jener als Flitterzellen bezeichneten Gebilde in.der Tiefe der Haut hervorgerufen werden, deren reflektiertes Licht den konstanten Untergrund bildet, über 
welchem sich das wechselnde Spiel der Chromatophoren vollzieht ${ }^{1}$ ). Am schärfsten lässt sich die Konstanz der Begrenzung der Innervationsgebiete dann feststellen, wenn Reizung eines Nerven die sogleich zu erwähnenden "Inseln" im fremden Innervationsgebiet ergibt, welche manchmal auffällig genau mit der Begrenzung eines weissen Flecks übereinstimmen. Natürlich kann die Konstanz des Resultates wiederholter Reizungen auch nur so lange anhalten, als der Nerv nicht etwa durch öftere starke Reizung geschädigt ist.

Dieser Befund von isolierten Innervationsbezirken bei künstlicher Reizung der Chromatophorennerven stimmt nun vollständig überein mit den Beobachtungen, die man über das Chromatophorenspiel des normalen Tieres machen kann. Die Tiere besitzen nämlich die Fähigkeit, die Chromatophoren an ganz bestimmten, ziemlich scharf begrenzten Stellen zu expandieren bzw. sie zusammenfallen zu lassen. Eine solche sehr auffallende lokale Expansion entsteht insbesondere als Schreckreflex ${ }^{2}$ ) an einer symmetrisch auf beiden Seiten des Rückens gelegenen Stelle. In Fig. 1 auf Taf. VI ist dieser ganz schwarz aussehende Fleck auf der linken Seite photographiert. Auf der rechten Seite fehlt er hier, weil sie gelähmt ist. Ferner beobachtet man häufig, besonders wenn sich zwei Sepien einander nähern, dass sie am Rücken eine Querbänderung von abwechselnd hellen und dunklen Streifen bekommen, welche "Zebrastreifung" in mehr oder weniger starker Ausbildung auch schon am ruhenden Tiere ausgebildet ist und zum Teil auf der bänderartigen Anordnung der schon erwähnten, weiss durch die Haut durchschimmernden, sehr reichlichen Flitterzellen beruht (vgl. Fig. 3 auf Taf. VI). Ruhende Tiere zeigen ferner häufig eine quer über die zusammengelegten Fangarme verlaufende Bänderung, welche in Fig. 2 u. 3 der Taf. VI schön zu sehen ist.

Manchmal beobachtet man einen nach vorn und nach der Seite zu sehr scharf absetzenden weissen Fleck über den Augen, von dem in Fig. 1 auf Taf. VI ein nicht einmal besonders drastisches Beispiel gibt. Zugleich mit diesem Fleck tritt in der Regel auch noch entweder eine weisse, fünfeckige, schildartige Stelle in der Mitte des Rückens oder auch eine sattelartig quer über den Rücken verlaufende

1) Vgl. dazu v. Ryn berk, Ergebn. d. Physiol. Bd. 5 S. 363 und anderwärts.

2) Ich habe dies öfters beobachtet, wenn ich rasch die Hand in die Nähe der Tiere hinbrachte oder dieselben beschattete. 
Bleichung. Kurz und gut, man kann schon durch die blosse Beobachtung des normalen Tieres unzweifelhaft feststellen, dass die Erregung im Chromatophorenapparat sich nicht allseitig ausbreitet, sondern auf gewisse, ziemlich gut begrenzte Stellen beschränkt bleibt.

Die dritte Tatsachenreihe, welche ebenfalls die isolierte Innervation einzelner Chromatophorenfelder beweist, ergiebt sich aus Lähmungsversuchen. Wie in der folgenden Abhandlung gezeigt werden soll, kann man durch Nervendurchschneidung eine abgegrenzte Lähmung bestimmter Chromatophorenbezirke herbeiführen, die sich in einer Ausbleichung der Haut kundgibt. Nach einiger Zeit färbt sich aber die gelähmte Hautstelle wieder dunkel infolge eines zunehmenden peripherogenen Tonus des Chromatophorenapparates. Auch dieser beschränkt sich auf das Gebiet des vorher durchschnittenen Nerven, wofür ein gutes Beispiel die Fig. 3 auf Taf. VI der folgenden Abhandlung zeigt. Man vergleiche dort auch die genaueren Erläuterungen zu diesem Versuch!

Nach dem Gesagten steht es unzweifelhaft fest, dass jedem Nervenstämmchen eine bestimmte abgrenzbare Zabl von Chromatophoren zugehört, welche unter normalen Umständen durch Reizung des Nerven in Erregung versetzt werden, dass demnach in diesem Sinne jeder Nerv sein besonderes Innervationsgebiet besitzt, über welches sein Eiufluss nicht hinausreicht. Dies ist nun, wie schon bemerkt wurde, nicht so zu verstehen, dass alle Chromatophoren eines geschlossenen Hautgebietes von einem und demselben Nerven aus in Erregung versetzt werden. Vielmehr sieht man häufig mitten im Gebiete der maximal expandierten Chromatophoren kleine Gruppen oder auch ganz vereinzelte, nicht expandierte Chromatophoren. Andererseits expandieren sich sehr häufig bei Reizung eines Nervenstammes ausser den in geschlossenen Bezirken - bis auf die eben erwähnten geringen Ausnahmen - zusammenliegenden Chromatophoren noch einzelne kleinere oder grössere Gruppen von Chromatophoren an anderer Stelle, und man kann dann in günstigen Fällen durch Präparation die zu diesen kleinen "Inseln in fremdem Gebiet" hinziehenden feinen Nervenfädchen darstellen. Der wichtigste Punkt aber, der hier zu beachten ist und auf den später noch genauer eingegangen werden soll, ist der, dass die Grenzen der Innervationsgebiete benachbarter Nerven derart übereinandergreifen, dass gewöhnlich die Chromatophoren eines Hautbezirkes von zwei nebeneinanderliegenden Nerven aus in Erregung versetzt : werden 
können. Wie stark dies Übereinandergreifen der Innervationsgebiete benachbarter Nerven ist, möchte ich durch Skizze 1 zeigen, welche die Grenzen der Hautdunkelung bei Reizung von vier nebeneinander liegenden Nervenbündeln wiedergibt, allerdings nur ungefähr. So war es fraglich, ob die Gebiete der Nerven 2 und 4 wirklich bloss aneinander anstossen, oder ob sie nicht doch vielleicht etwas übereinandergreifen. Dagegen trat sehr deutlich die Inselbildung bei Reizung von Nerv 1 und 4 im geschlossenen Gebiete von Nerv 2 hervor, und besonders drastisch ist die Deckung des Innervationsgebietes von Nerv 3 teils durch Nerv 2, teils durch Nerv 4.

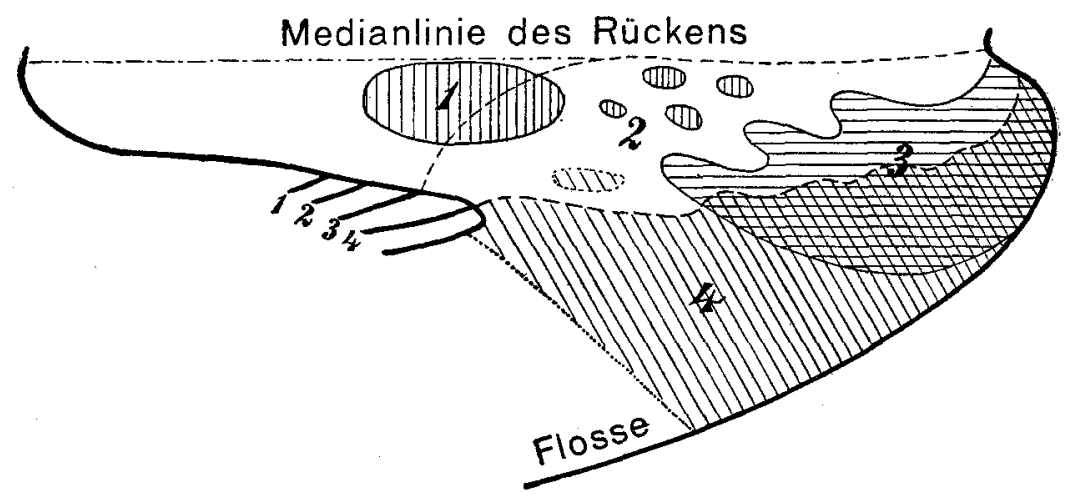

Fig. 1. Grenzen der Innervationsbezirke von vier Hautnerven der linken Seite. Sepia offic. Das Innervationsgebiet des sehr dünnen Nerven 1 ist vertikal schraffiert und besteht aus einer geschlossenen Partie in der Mitte des Rückens und mehreren kleinen Inseln weiter hinten, viel mehr, als eingezeichnet sind. Das Gebiet des Nerven 2 ist weiss gelassen und durch eine gestrichelte Linie abgegrenzt; das Gebiet des Nerven 3 ist horizontal, das des Nerven 4 schräg schraffiert. „Die Markierung der innervierten Hautpartien wurde dadurch erleichtert, dass nach einer starken Reizung die Hautstelle lange

Zeit bleich, wie gelähmt erscheint." (Protokoll vom 16. März 1904.)

Wenn wir nun fragen, wie sich denn die Existenz besonderer Innervationsgebiete für jeden Nerven mit der Annahme einer Erregungsleitung in einem geschlossenen peripheren Nervennetze vereinbaren liesse, so kommen wir zu demselben Resultate, wie in den beiden vorhergehenden Abschnitten. Die letztere Hypothese lässt sich ganz offenkundig nur dann festhalten, wenn man hinzufügt, die Erregung pflanze sich im Nervennetz mit sehr starkem Dekrement fort. $O b$ ein solches Dekrement wirklich vorhanden ist, das scheint nun bei den Chromatophoren einer Entscheidung zugänglich zu sein. Denn hier können wir ja bis auf die einzelne Chromatophore genau die Grenze der Innervation feststellen. Es fragt sich also, ist diese 
Grenze bei maximaler Nervenreizung eine ganz scharfe oder schattiert sie sich allmählich von einer maximalen Erregung in der Mitte nach der Grenze gegen die nicht erregten Gebiete zu ab.

Bei Beobachtung unter der Lupe stellt sich nun die Grenze als eine ziemlich scharfe, stellenweise sogar ganz schroffe dar. Ich zitiere aus meinen Protokollen (ausführlicher ist das Protokoll unten S. 398 unter Nr. 4 mitgeteilt): "Mit der Lupe die Grenze des Innervationsbezirkes beobachtet. An der Grenze entlang sieht man ganz wenig submaximal gereizte Chromatophoren ..." „Sehr interessant ist eine Stelle, wo ringsherum maximal expandierte Chromatophoren und dazwischen eine Gruppe von etwa fünf gänzlich unerweiterten, an der Grenze befinden sich zwei submaximal expandierte ... Stellenweise sieht man mitten im Gebiete der maximal expandierten Chormatophoren vereinzelte ganz unerweiterte."

Dies Resultat ist nun natürlich nach der einen wie nach der anderen Seite hin verwendbar. Man kann sagen, die oft ganz abrupte Scheidung zwischen maximal expandierten und gar nicht erweiterten Chromatophoren (besonders bei den einzelnen eingestreuten) zeigt die strenge Isolierung der Ausbreitungsbezirke der einzelnen Nerven. Man kann aber auch auf die wenigen submaximal expandierten Chromatophoren hinweisen, um eine, wenn auch nur ganz unbedeutende, Ausbreitung der Erregung in einem peripheren Nervennetz zu begründen. Die Anhänger der ersterwähnten Meinung könnten dem gegenüber wieder geltend machen, was unten noch näher begründet werden soll, dass nämlich diese Chromatophoren von zwei Nerven aus innerviert sind und vielleicht die submaximale Expansion darauf beruht, dass von den betreffenden Nerven nur wenige Endfädchen zur Chromatophore hinziehen. Endgültig entscheidend nach der einen oder anderen Richtung ist also das Resultat nicht. Trotzdem ist es von Bedeutung. Es erhellt nämlich daraus ganz klar, dass selbst, wenn ein peripheres kontinuierliches Nervennetz vorhanden sein sollte, die Erregung in ihm sich so wenig fortpflanzt, dass praktiseh fast dasselbe erreicht wird, als ob die Endnetze der einzelnen Nervenfasern auch histologisch gänzlich voneinander gesondert wären. Das periphere Nervennetz würde sich demnach, wenn es überhaupt existiert, in einer sehr wesentlichen Eigenschaft von den Nervenstämmchen unterscheiden. Diese können zwar beim Absterben und nach Behandlung mit manchen Giften (vgl. die folgende Abhandlung) 
ein deutliches Dekrement der Erregung zeigen, aber auch dann kaum in dem hohen Grade, wie es in dem peripheren Netze der Fall sein müsste. Auch ist der Einwand ganz unhaltbar, dass sich dieses Dekrement im peripberen Nervennetz erst nach dem Tode als Absterbeerseheinung eingestellt haben sollte. Dagegen sprechen zunächșt die oben S. 388 angeführten Beobachtungen am intakten Tiere, und ferner das abweichende Verhalten eines wirklichen Dekrementes im peripheren Nervengeflecht, das sich als Absterbeerscheinung tatsächlich einstellen kann. Ich habe das besonders schön an einem Tiere beobachten können, das von Triest nach Innsbruck transportiert und auf dem Wege gestorben war. Die Reizung der frei präparierten Nerven gaben hier keine Expansion der Chromatophoren mehr offenbar wegen des zu starken Dekrementes der Erregungsleitung -, wohl aber konnte man beim Aufsetzen der Elektroden auf die Haut noch ausgebreitete Expansion der Chromatophoren auslösen. Diese grenzte sich gegen die bleichen Seitenpartien der Haut zu ganz gut $a b$, trotzdem, wie gesagt, ein Dekrement der Erregungsleitung in den peripheren Nerven zweifellos vorbanden war, was aus der ganz ungewöhnlich grossen Menge submaximal expandierter Chromatophoren gegen die Grenze zu hervorging. Hier sieht mạn nun (auch an photographischen Aufnahmen in natürlicher Grösse) ganz deutlich den grossen Unterschied zwischen der. offenkundigen allmählichen Erregungsabnahme mit der Entfernung von der gereizten Stelle und dem ganz andersartigen steilen Abbrechen der Erregung an der Grenze der nicht innervierten Partie. Besonders an einem isolierten, durch unerregtes Gebiet von der Reizstelle getrennten Flecke war dies auffällig. Nebenbei bewies das Auftreten dieses Fleckes sowie die Anwesenheit isolierter expandierter Chromatophoren im sonst blassen seitlichen Teile der Haut, dass die Abnahme der Erregung mit der Entfernung von der Reizstelle nicht etwa einfach aus der Abnahme der Stromschleifen zu erklären ist; welche die Chromatophoren direkt reizen, sondern dass wirklich nahe an den Elektroden liegende Nervenbündel gereizt sein müssen.

Eine weitere Probe auf die Anwesenheit eines mit Dekrement leitenden peripheren Nervennetzes müsste der Vergleich submaximaler mit maximalen Reizungen liefern, bzw. die Vorgänge bei allmählicher Verstärkung der Reizung von der Schwelle an. Für den Fall, dass die Nervenfasern in der Peripherie in ein kontinuierlich ausgebreitetes Nervennetz überginge, welches eine gleichmässsige Ausbreitung der 
Erregung nach allen Richtungen, aber mit rascher Abnahme der Erregungsgrösse gestattete, sollte man erwarten, dass bei submaximaler Reizung die submaximale Erregung auf die nächste Umgebung der Einmündungsstelle der Nervenfaser in das Nervennetz beschränkt bliebe, dass aber bei weiterer Verstärkung der Reizung mit der Zunahme der Erregungsgrösse bis zum Maximum auch eine weitere Ausbreitung der Erregung bis zu einem Maximum erfolgte, Gibt es dagegen kein peripheres Nervennetz, sondern sind die Endausbreitungen jedes Neurons von dem des anderen isoliert, so würde sich sowohl bei submaximaler als auch bei maximaler Reizung die Erregung stets auf das gleiche Gebiet erstrecken müssen. Der Unterschied zwischen submaximaler und maximaler Reizung würde sich dann bloss in der Stärke der Erregung äussern können.

Tatsächlich liegen die Verhältnisse nun freilich ziemlich verwickelt, aber doch so, dass sie sich mehr dem Schema der zweiten Annahme nähern. Als erster Erfolg der Reizung mit ganz schwachen Strömen tritt nur selten eine Expansion aller Chromatophoren auf; die bei maximaler Reizung betroffen werden, sondern es expandieren sich gewöhnlich bloss einzelne Flecken, Streifen oder kleinere Gruppen, die oft durch unerregte Felder voneinander getrennt sind, und sich zunieist gleich von vorne herein mit demselben Grade von Schärfe gegen die unerregte Umigebung absetzen, wie es oben für die maximale Reizung geschildert wurde. Schiebt man nun während der Reizung die sekundäre Spule gegen die primäre zu vor, so vermehren und verbreitern sich diese Flecken und Flächen, aber nicht der allmählichen Zunahme der Reizstärke entsprechend in all mählichem Wachstum, sondern sprungw eis e; d. h. bei gleichmässigem Vorschieben bleibt zunächst eine Zeitlang alles unverändert, und dann expandieren sich ganz plötzlich die Chromatophoren eines weiteren Gebietess. Das Spiel kann sich beim weiteren Vorschieben der selsundären Spirale mehrmals wiederholen. Der Vorgang vollzieht sich auch nicht etwa immer so, dass sich die Erregung bei Verstärkung der Reizung von einer Stelle aus, wenn auch sprungweise, so doch auf die nächste Umgebung der zuerst ergriffenen Stelle ausbreitet, sondern es kommt auch vor, und das ist für die Beurteilung der Sachlage besonders bemerkenswert, dass bei Reizung eines etwas dickeren Nervenstämmehens zuerst ein dunkler Fleck an einer Stelle auftritt, bei Verstärkung der Reizung ein anderer Fleck dazu kommt, 
der von dem ersten durch einen grösseren nicht erregten Zwischenraum getrennt ist.

Die sprunghafte Vergrösserung des Erregungsgebietes deutet schon darauf hin, und das Auftauchen neuer von den alten getrennter Flecken bei allmählicher Verstärkung der Reizung beweist es geradezu, dass diese Art der Ausbreitung der Erregung durch ein allmähliches Fortschreiten derselben in einem kontinuierlichen Nervenendnetz nicht erklärt werden kann. Vielmehr muss man auf zwei andere Umstände rekurrieren. Es kann nämlich zunächst eine verschiedene Reizbarkeit der nebeneinander im Nervenstamm liegenden Nervenfasern vorhanden sein, deren Erklärung am allereinfachsten in der unvermeidlichen Schädigung bei der Präparation (Zerrung, Querschnittsanlegung durch Wegschneiden von Nebenästchen) gesucht werden darf ${ }^{1}$ ). Ein direkter Beweis dafür liegt in folgendem Experiment. Reizt man einen lang herauspräparierten Nerven direkt an der Eintrittsstelle ins Unterhautzellgewebe mit so starken Strömen, dass sie eben für eine maximale Erregung der Chromatophoren des ganzen Innervationsgebietes ausreicht, und fährt man dann bei unverändertem Rollenabstand mit den Elektroden am Nerven entlang bis zum Querschnitt zurück, so wird man in der Mehrzahl der Fälle sehen, dass von hier aus eine fleckweise Chromatophorenerregung zu erzielen ist, was natürlich leicht begreiflich ist, weil die von der Nähe des Querschnittes ausgehende Nervenerregung alle geschädigten Stellen passieren muss.

Der zweite in Betracht kommende Einfluss ist der der Stromdichte, die ja in den Nervenfasern, welche den Elektroden unmittelbar anliegen, am grössten, in den entfernteren aber entsprechend geringer sein muss. Besonders bei etwas dickeren Nervenbündeln

1) Es ist allerdings auch sehr wohl möglich, dass schon vor der Präparation eine verschiedene Reizbarkeit der nebeneinander liegenden Nervenfasern vorliegt. Darauf könnte die mehrfach gemachte Erfahrung hindeuten, dass sich bei eben überschwelliger Reizung zuerst jene Streifen ausbilden, welche der oben S. 388 erwähnten natürlichen „Zebrastreifung" des Tieres entsprechen. Ganz gewiss ist aber eine solche Differenz der Reizbarkeit zwischen dem neuromuskulären peripheren Apparat der Chromatophoren und des Flossenmuskels vorhanden. Ich habe fast immer beobachtet, dass die Flossenmuskulatur anfangs schon auf viel schwächere Nervenreizung anspricht als die Chromatophorenmuskeln. Dagegen verschwindet aber bei wiederholter Reizung ihre Erregbarkeit bald (wegen O-Mangel in die Tiefe?), während die der Chromatophoren noch lange erhalten bleibt. 
musste bei den verwendeten Reizelektroden (Platindrähte, die nur etwa $2 \mathrm{~mm}$ voneinander entfernt waren) die Differenz der Stromdichte sehr beträchtlich sein. Ein exakter Beweis für die Mitwirkung dieses ja für die Nerven der Wirbeltiere wiederholt eingehend erörterten Faktors ist mir aber nicht gelungen. Bei dem einfachsten Versuche: Drehen des Nerven auf den Elektroden, sind Verschiebungen des Nerven unvermeidlich, somit der Einfluss des zuerstgenannten, jedenfalls sehr wesentlichen Faktors nicht völlig ausgeschlossen.

Dass sich ausser den beiden erörterten Faktoren, welche die sprunghafte Erweiterung des Erregungsgebietes erklären, noch ein allerdings sehr mässiges Fortschreiten der Erregung in einem Nervenendnetz an der Ausbreitung der Erregung beteiligt, ist vielleicht möglich, kann aber nicht sicher bewiesen werden. Jedenfalls würde diese Ausbreitung, selbst wenn sie vorhanden wäre, gegenüber der sprunghaften Zunahme des Erregungsgebietes nur eine untergeordnete Rolle spielen.

Kompliziert werden die Verhältnisse bei Reizung mit schwachen Strömen noch durch drei weitere Umstände. Erstens nämlich kann man gelegentlich eine Art Ermüdung des Chromatophorenapparates konstatieren, insofern, als bei eben überschwelligen Strömen, die Erregung nur zu Beginn der tetanischen Reizung vorhanden ist und noch während der Dauer der Reizung versehwindet. Bei Wiederholung der Reizung kann dann auch dieser Anfangseffekt (vergleichbar dem "Anfangstetanus") vollends ausbleiben. $\mathrm{Ob}$ es sich dabei um eine "Ermüdung durch Leitung" oder um eine Herabsetzung der Reizbarkeit bloss an der Reizstelle handelt, muss ich dahingestellt sein lassen.

Noch weitaus interessanter ist eine andere Erscheinung, die ich sehr oft beobachtet habe, und zu der ich ein vollständiges Analogon bei anderen reizbaren Gebilden nur ganz ausnahmsweise gesehen habe ${ }^{1}$ ). Es kam nämlich gar nicht selten vor, dass die Erregung der Chromatophoren bei Reizung des Nerven mit schwachen Strömen dem Reizbeginn erst nach einer merklichen Latenzzeit nachhinkte. Dies war auch der Fall, wenn der Nerv während der Reizung ganz ruhig und unberührt auf den Elektroden lagerte, so dass der Gedanke, dass vielleicht eine Verschiebung des Nerven auf 
den Elektroden, wodurch etwa erst nachträglich eine besser reizbare Nervenstelle getroffen würde, ganz ausgeschlossen ist. Dazu kommt, dass in solchen Fällen sehr oft die Erregung auch einige Zeit, ausnahmsweise sogar ziemlich lange über das Ende der Reizung hinaus anhält ${ }^{1}$ ). Reizt man gleich darauf mit starken Strömen, so entsteht und verschwindet die Erregung sofort mit Beginn und Ende der Reizung, und nachher tritt sogar eine Art Lähmung auf, d. h. wenn vor der Reizung infolge des Liegens an der Luft eine mässige Expansion der Chromatophoren eingetreten war, so macht sie nach der Reizung im Innervationsgebiete des Nerven einer völligen Ausbleichung Platz. Ich habe diese letztere Erscheinung, die in der Frklärung zu Fig. 1 schon erwähnt wurde, immer benützt, wenn ich eine Hautstelle, die sich in schwacher Dauererregung befand, für den Versuch bleich bekommen wollte.

Das Nachhinken der Erregung zu Beginn und am Ende der Reizung ist demnach ein Charakteristikon schwacher, eben überschwelliger Reizungen. Es stimmt daher zu den obigen Ausführungen, dass man bei etwas stärkeren Strömen mitunter mit der Reizung synchron, Erregung jener Stellen beobachtet, welche schon auf schwächere Reize angesprochen hatten, während die neu hinzugekommenen Erregungsgebiete nachhinken, zum Beweis dafür, dass für sie die Reizung eben überschwellig ist. Man kann dann zu Beginn der Reizung sofortige Dunkelung eines Gebietes und Nachschlagen der unmittelbaren Umgebung beobachten, was oberflächlichen Beobachtern als Wanderung der Erregung imponieren könnte.

Das Nachhinken der Erregung nach schwacher Reizung ist durchaus nicht immer vorhanden. Worauf es beruht, kann ich nicht sagen. Es ist in dieser Hinsicht vielleicht bemerkenswert, dass ich bei dem Versuche, die Chromatophoren durch Alkohol zu lähmen, nach Applikation einer 10\% igen Lösung von Alkohol in Seewasser auf die Haut gelegentlich ein auffallend starkes Nachhinken der Alkoholstelle beobachtet babe, während die Umgebung der Reizung synchron reagierte. Wenn man in diesem Fall den Nerv nur ganz kurze Zeit tetanisierte, so schwärzte sich die Umgebung, nicht aber die Alkoholstelle! Sowie aber

1) Bei Reizung der pupillenerweiternden Nerven sieht man gelegentlich auch ein solches Nachhinken nach Schluss der Reizung, nämlich eine ganz allmähliche Verengerung der Pupille, die Langley (Journ. of Physiol. vol. 31 p. 247. 1904) beschrieben hat, und die ich aus eigener Erfahrung bestätigen kann. Ein Nachhinken zu Beginn der lieizung habe ich aber hier bisher nur einmal bei Reizung von Vorderwurzelbündeln des 2. Thorakalnerven bei einer sehr stark mit Chloralose vergifteten Katze gesehen. 
die Reizung so lange dauerte, dass die Alkoholstelle ebenfalls dunkel wurde, so blasste sie auch später aus, hinkte also auch am Ende der Reizung nach. Später, als die Alkoholwirkung noch stärker ausgesprochen war, blieb die Mitte der vergifteten Stelle während der Reizung dauernd hell, von der Umgebung aber breitete sich die Erregung während der Reizung infolge des Nachhinkens anscheinend langsam in die Alkoholstelle hinein aus. Vielleicht wäre hier ein Weg gegeben, die Erscheinung weiter zu analysieren.

Eine dritte Erscheinung, die, so interessant sie an sich ist, bei den jetzt in Betracht kommenden Versuchen lediglich störend wirkt, ist das "Pulsieren" der Chromatophoren ${ }^{1}$ ). Es kommt vor, dass einzelne Chromatophoren oder ganze Gruppen während der Dauer der Reizung nicht gleichmässig expandiert bleiben, sondern ziemlich rhythmisch sich abwechselnd stärker expandieren und dann wieder etwas retrabieren. In solchen, übrigens nicht sehr bäufigen Fällen ist es natürlich ziemlich schwer, sichere Beobachtungen über die Ausdehnung des Innervationsgebietes anzustellen, besonders wenn in der Umgebung (infolge der Reizung durch das Liegen an der Luft?) einzelne Chromatophoren ebenfalls pulsieren.

Zur Illustration für das Gesagte füge ich hier einige besonders charakteristische Protokollstellen, soweit als möglich wörtlich, ein (geändert sind bloss die nur mir verständlichen Abkürzungen, die weiter ausgeführt werden), welche, unter dem frischen Eindruck des eben Gesehenen rasch hinstenographiert, vielleicht besser als langatmige Beschreibungen das Schlagende mancher Experimente lebendig machen. Alle Protokolle beziehen sich auf Sepia officinalis.

1. Versuch vom 14. April 1904. Reizung des Nerven etwa $1^{1 / 2}$ Stunde nach der Tötung des Tieres. R.-A. $\left.{ }^{2}\right) 20 \mathrm{~cm}$ : Anfangstetanus einer bestimmten Stelle. R.A. $18 \mathrm{~cm}$ : Es schwärzt sich dieselbe Stelle, während der ganzen Reizdauer anhaltend. R.-A. $15 \mathrm{~cm}$ : Dazu kommen noch einige Chromatophoren weiter hinten, ausserdem bildet sich eine wunderschöne Hautfalte durch Kontraktion des Hautmuskels. R.-A. $12 \mathrm{~cm}$ : Die hinteren Chromatophorengruppen expandieren sich viel schöner und stärker. Bemerkung zu R.-A. 15 und $12 \mathrm{~cm}$ : Anscheinend erschlaffen jene Stellen, welche erst bei anhaltender Reizung mit etwas stärkeren Strömen später (d. h. nachhinkend) hinzukommen, wie hier bei R.-A. $15 \mathrm{~cm}$ die hinteren Chromatophorengruppen, auch nach Schluss der Reizung später. Von R.-A. 12 bis $6 \mathrm{~cm}$ ändert sich an der Ausbreitung der Innervation nichts mehr.

2. Versuch rom 20. April 1904. Tier war schon in der Nacht gestorben, Nervenreizung erst nachmittags nach 4 Uhr. Reizung eines Nerven "ganz unten", d. h. unmittelbar vor seinem Eintritt ins Unterhautgewebe zunächst mit R.-A. 17, wobei sich nur einige nahe Chromatophorengruppen expandieren, dann bei Ver:stärkung der Reizung immer weitere Gruppen bis zu R.-A. $6 \mathrm{~cm}$. Es kommt vor,

1) Vergleiche darüber Steinach, Studien über die Hautfärbung und den Farbenwechsel der Cephalopoden. Pflüger's Arch. Bd. 87 S. 5. 1901.

2) R.-A. = „Reizung mit Rollenabstand“. 
dass die neu hinzukommenden Gruppen nach Schluss der Reizung etwas länger expandiert bleiben und langsamer erschlaffen als die nahen Gruppen, aber das. geschieht nicht immer; einigemale auch, dass sie erst nach Beginn der Reizung. hinzukommen, so dass die Dunkelung absatzweise erfolgt, im Doppelschlag, zuerst die nahe Hauptgruppe, dann die benachbarte Nebengruppe. Bei etwas schwächerer Reizung kommt letztere gar nicht, bei etwas stärkerer Reizung sofort hinzu: bei: R.-A. 9,0 cm gar nicht, bei $8,7 \mathrm{~cm}$ mit Absatz, bei R.-A. $8,5 \mathrm{~cm}$ sofort. (Das Experiment zeigt Erscheinungen, welche auf schlechte Reizbarkeit des lange Zeit nach dem Tode untersuchten Nerven hindeuten.)

3. Versuch rom 21. April 1904. Präparation und Reizung der Nerven sofort, nachdem das Tier getötet worden war. Erster Nerv: Reizschwelle für die Chromatophoren bei R.-A. $21,0 \mathrm{~cm}$. Dabei tritt nur fleckweise Dunkelung auf. Sehr schöne Ermüdung: Zunächst kommen die Fleckchen erst einige Zeit nach Beginn der Reizung, dann - bei wiederholter Reizung - immer später, schliesslich verschwinden sie bei läugerer Reizung noch während der Reizdatuer und bei neuerlicher Rejzung nach kurzer Pause kommt gar nichts mehr. Darauf bei R.-A. $20,5 \mathrm{~cm}$ sind die Fleckchen zunächst prompt (d. h. sofort bei Beginn der Reizung) da, bei Wiederholung der Reizung hinken sie dann dem Reizbeginn. einige Zeit nach, verschwinden aber prompt bei Schluss der Reizung. Zweiter Nerv: Bei R.-A. 16,0 cm expandieren sich einzelne Chromatophoren, bei R.-A. $15,0 \mathrm{~cm}$ mehrere Chromatophoren, bei R.-A. $14,0 \mathrm{~cm}$ das ganze Gebiet. Ich. schiebe von R.-A. 16,0 cm während anhaltender Reizung langsam vor. Bis R.-A. $12,0 \mathrm{~cm}$ erfolgen noch kleine Änderungen, die sich hauptsächlich darauf beziehen, dass zuerst sehr schwankende Partien dauernd dunkel werden, anscheinend Ermüdung ausgeglichen wird. Von R.-A. 12 bis $7 \mathrm{~cm}$ erfolgt keine Verschiebung: der Grenze mehr. Dreimal wiederholt! Dritter Nerv: Reizschwelle für den Hautmuskel bei R.-A. 23,0 cm, für die Chromatophoren bei $19,0 \mathrm{~cm}$. Bei $19,0 \mathrm{~cm}$ grossartig: Zunächst verschiebt sich die Haut, dann expandieren sich langsam. die Chromatophoren, nach Schluss den Reizung bleiben sie noch eine ganze Weile expandiert und retrahieren sich dann ganz allmählich. Die Stellen sind. nachher ganz blass, bilden kleine weisse Flecken und Streifen. Sekundäre Spirale vorgeschoben, um $12 \mathrm{~cm}$ herum kommen noch einige Gebiete dazu, dann vorgeschoben bis $2,5 \mathrm{~cm}(!)$ : unveränderte Grenze für die Chromatophoren, bloss der Hautmuskel kontrahiert sich immer mächtiger.

4. Versuch vom 25. April 1906. Nerv 41/2 Stunden nach der Tötung. des Tieres untersucht. Bei starker Reizung des Nerven mit der Lupe die Grenze. des Innervationsbezirkes beobachtet. An der Grenze entlang sieht man ganz. wenig submaximal gereizte Chromatophoren (die beigefügte Skizze zeigt zwei solche). Gelegentlich sieht man bei anhaltender Reizung, dass einige Zeit nach Beginn derselben eine Gruppe von früher nicht expandierten Chromatophoren aufschiesst, dann aber, eventuell nach nochmaligem Aufpulsieren wieder verengt bleibt. Eine Gruppe beobachtet, welche während der Reizung viele Male pulsierte. Dann kommt es vor, dass an der Grenze gelegene Chromatophoren in zwei Absätzen nacheinander sich expandieren und über das Ende der Reizung hinaus erweitert bleiben. Das könnte aussehen wie eine kurze Fortpflanzung der Erregung durch ein Nervennetz. Sehr interessant ist eine Stelle, wo. 
xingsherum maximal expandierte Chromatophoren und dazwischen eine Gruppe von etwa fünf gänzlich unerweiterten, an der Grenze befinden sich zwei.submaximal expandierte, eine davon pulsierend. Stellenweise sieht man mitten im Gebiete der maximal expandierten Chromatophoren vereinzelte ganz unerweiterte. Auch sieht man innerhalb des geschlossenen Gebietes von expandierten Chromatophoren gelegentlich zu Beginn der Reizung Expansion in zwei Etappen: einige Chromatophoren hinken nach. Bei ganz kurzdauernden Reizungen sieht man besonders deutlich das Nachhinken der Erregung hinter der Reizung.

Nach allem, was mitgeteilt wurde, ist also bei den Chromatophoren unter normalen Umständen eine allseitige Fortleitung der Frregung in der Peripherie von irgendwelchem Belange gänzlich ausgeschlossen. Dass damit auch die Existenz eines peripheren Nervennetzes völlig ausgeschlossen ist, kann man natürlich zunächst noch nicht sagen. Man muss ihm nur die besondere Eigenschaft zuschreiben, dass es die Erregung nur auf allerkürzeste Strecken mit sehr hohem Dekrement fortleite. Die nunmehr noch zll besprechende Tatsache lässt, vielleicht sogar manchem die Hypothese, dass wirklich ein geschlossenes Nervennetz in der Peripherie vorbanden ist, einigermaassen plausibel erscheinen. Wir baben oben schon davon gesprochen, dass die Innervationsgebiete benachbarter Nerven stark übereinandergreifen. Da wir aber ausserdem gesehen haben, dass innerhalb der Gebiete maximaler Expansion sehr häufig noch einige unerregte Chromatophoren ủbrig bleiben, so könnte man auf die Meinung kommen, dass dies Übereinandergreifen der Innervationsgebiete sich bloss darauf bezieht, dass die verschiedenen Chromatophoren eines Hautgebietes von verschiedenen Nerven versorgt würden, aber jede Chromatophore nur von einer Nervenfaser her. Bei einer genaueren Beobachtung des Reizerfolges lässt sich aber diese Meinung nicht aufrechterhalten. Man sieht zunächst häufig genug bei Reizung zweier benachbarter Nerven an einer und derselben Stelle eine so starke Expansion der Chromatophoren, dass der Gedanke, durch die übereinandergeschobenen Ränder der expandierten Chromatophoren würden vielleicht einige nicht expandierte bloss verdeckt, von vornherein sehr unwahrscheinlich wird. Vielmehr ist die Annahme nicht zu umgehen, dass ein und dieselbe Chromatophore von zwei verschiedenen Nerven her in Erregung versetzt werden kann. Auf völlig exakte und ganz einwandfreie Weise konnte ich diesen Satz beweisen durch gleichzeitige Reizung zweier benachbarter Nerven und Beobachtung des Grenzgebietes. Die Erregung des einen Nerven, nennen wir ihn $a$, war eine dauernd gleichmässige, hervor- 
gerufen durch chemische Reizung (mit $1 / 40$ norm. $\mathrm{NaOH}$ ). Der daneben liegende Nerv gab, wenn er mit $20-16 \mathrm{~cm}$ R.-A. gereizt wurde, eine ins Gebiet des anderen hinübergreifende Expansion von Chromatophoren, und zwar expandierten sich dabei nicht bloss vereinzelte im Gebiete des Nerven $a$ befindliche, bisher noch nicht erregte Chromatophoren, sondern es wurde auch die Expansion mehrerer, an der Grenze befindlicher Chromatophoren, welche bei Reizung des Nerven $a$ allein nur halb oder zu dreiviertel expandiert waren, maximal. Ich habe diese Beobachtung unter der Lupe mehrmals wiederholt und kann sie als ganz zuverlässig bezeichnen. Um ganz sicher zu gehen, dass die Erregung der halb oder zu dreiviertel expandierten Chromatophoren durch die chemische Nervenreizung hervorgerufen worden war, schneide ich darauf den Nerv $a$ unterhalb der Reizstelle durch. Daraufhin verschwindet die Erregung sämtlicher Chromatophoren seines Gebietes, alle früher expandierten retrahieren sich. Es kann demnach kein Zweifel darüber bestehen, dass eine Chromatophore von zwei Nerven her in Erregung versetzt werden kann.

Dies Resultat lässt sich nun allerdings mit jeder der beiden einander entgegenstehenden Meinungen vereinbaren. Man kann entweder annehmen, dass die an jeder einzelnen Chromatophore angreifenden Teiläste eines Neurons nur unter sich, nicht aber mit denen eines Nachbarnerven zur Bildung der in der histologischen. Untersuchung beschriebenen Guirlanden sich vereinigen. Dann aber muss man allerdings hinzufügen, dass zu den balb oder zu dreiviertel expandierten Chromatophoren wahrscheinlich nur wenige Nervenzweige des betreffenden Neurons hinzutreten. Da in der Regel bei ganz frischen Chromatophoren eine gleichzeitige Kontraktion aller, nicht eine isolierte Kontraktion einzelner Radiärmuskeln beobachtet wird, so würde eine Frregung, die an irgend einer Stelle von ganz vereinzelten Nervenzweigchen auf einzelne Radiärfasern übertragen. wird, wahrscheinlich auf alle anderen Radiärmuskeln übergehen ${ }^{1}$ ), aber entsprechend der kleinen Angriffsfäche nicht maximal sein. Selbst wenn eine und dieselbe Chromatophore von zwei verschiedenen. Neuronen aus jedesmal zu maximaler Expansion veranlasst werden kann, wenn man also nach dem Vorigen voraussetzen müsste, dass

1) Dass eine zunächst nur lokal durch Reizung einzelner Radiärfasern gesetzte Erregung sich auf den garzen Muskeiapparat der Chromatophore ausbreitet, ist auch Steinach's Meinung (Pfïüger's Ařch. Ed. 87 S. 18). 
von jedem Neuron sehr viele Endzweige zu ihr hinziehen, selbst dann würde man noch immer ganz wohl annehmen dürfen, dass die Endzweige jedes Neurons gesondert bleiben, denn wir haben ja bei der histologischen Untersuchung gesehen, dass in der Tat an jeder einzelnen Radiärfaser mehrere Nervenfädchen gesondert beobachtet werden können.

Auf der anderen Seite würde man, unter Voraussetzung eines kontinuierlichen peripheren Nervennetzes, bloss anzunehmen brauchen, dass gewisse Stellen des Netzes mehreren Nervenfasern in dem Sinne gemeinsam angehören, als die Erregung von jeder einzelnen dieser Nervenfasern aus auf dieselbe Stelle des Netzes überzugehen vermag, was ja in keinem Widerspruch steht zu der oben gezogenen Folgerung, dass schliesslich in den Endfasern selbst die Erregung sich nur auf kürzeste Strecken mit starkem Dekrement fortpflanzt. Überhaupt wird man gegen keine dieser Annahmen einen völlig triftigen Einwand erheben können. Welcher man sich zuneigt, wird daher mehr von anderweitigen Überlegungen abhängen. Ich muss gestehen, dass mir gegenwärtig die ersterwähnte Ansicht doch etwas gezwungener vorkommt, und dass mir auch aus den schon in der histologischen Untersuchung mitgeteilten Gründen die Annahme eines allseitig geschlossenen Endnetzes einfacher und plausibler vorkommt. Aber das ist eine blosse Meinungssache, die durch das Gewicht auch nur einer einzigen entgegenstehenden Tatsache sofort umgestossen werden kann.

\section{B. Das "Wolkenwandern".}

Unter gewissen abnormen Bedingungen tritt nun allerdings auch an den Chromatophoren, und zwar nicht bloss bei Sepia, sondern auch bei Eledone und Oktopus, ein Phänomen auf, das auf den ersten Blick anscheinend nicht anders erklärt werden kann als durch eine Fortpflanzung der Erregung in einem peripheren kontinuierlichen Leitungsnetz. Die Erscheinung ist von Steinach ${ }^{1}$ ) eingehend an der Haut von Eledone und Oktopus studiert worden und von ihm als Wellenspiel bezeichnet worden. Ich beschränke mich hier hauptsächlich auf die Verhältnisse bei Sepia officinalis. Die analoge Erscheinung bei Eledone moschata habe ich zwar zur eigenen Information ebenfalls schon studiert, gedenke aber eventuell erst später

1) Pflüger's Arch. Bd. 87 S. 18 ff. 1901. 
einmal darauf zurückzukommen. Es handelt sich darum, dass unter gewissen Umständen an einer Stelle der sonst bleichen Haut einige Chromatophoren sich expandieren, so dass dort ein dunkler Fleck entsteht, der nun in ganz unregelmässiger Richtung, jedoch ganz ausnahmslos in kontinuierlichem Zuge ohne Überspringung einer Zwischenstelle vom Ursprungsort aus weiter wandert. Nur diese Erscheinung verstehe ich unter "Wellenwandern" und fasse also diesen Begriff enger als den von Steinach (l. c.) aufgestellten des „Wellenspiels" der Chromatophoren. Steinach rechnet zum Wellenspiel auch gewisse Phänomene am lebenden normalen Tiere. Man sieht nämlich gelegentlich bei Eledone moschata wie einen Schatten ganz rasch über die Haut hinhuschen. Ich möchte diese Erscheinung, als offenbar vom Zentralnervensystem aus hervorgerufen, streng sondern von dem langsamen Wellenwandern, das unter gewissen Umständen an der vom Zentralnervensystem isolierten Haut zu beobachten ist. Nicht mit einbezieben möchte ich ferner den raschen, ganz unregelmässigen Wechsel der Chromatophorenexpansion bei der lebenden Eledone, den Steinach (l. c.) beschreibt, sowie eine Erscheinung, die bei lebenden Sepien mitunter auftritt, wobei die Chromatophoren in höchst unregelmässigen Gruppen pulsatorisch sich abwechselnd expandieren und wieder zusammenfallen, so dass eine Erscheinung auftritt, welche man am ehesten mit dem ganz unregelmässigen Glitzern einer sonnenbeschienenen, leicht vom Winde „gekräuselten" Wasserfläche vergleichen könnte.

Das Wolkenwandern oder Wellenspiel im engeren Sinne, so wie ich es abgrenze, ist demnach keine normale Erscheinung. Es tritt nur unter gewissen Versuchsbedingungen auf, und auch dann beschränkt es sich bei Sepia officinalis (nicht bei Eledone und Oktopus!) fast nur auf solche gelähmte Hautpartien, deren Nerven einige Tage vor dem Versuche durehschnitten worden waren. Nicht vorher gelähmte, in diesem Sinne also "normale" Hautpartien zeigen (bei Sepia!) unter den gleichen Bedingungen höchstens ein rudimentäres, bloss auf ganz kurze Strecken sich ausbreitendes Wolkenwandern.

Will man das Wolkenwandern in der herrlichsten Weise beobachten, so vergifte man entweder ein lebendes Tier, hei dem eine Mantelhälfte seit längerer Zeit gelähmt ist oder noch einfacher ein dem eben getöteten Tiere entnommenes Stück vorher gelähmter Haut mit Koblensäure! Ich habe die Versuche in der Regel so angestellt, dass ich von Sepien, deren eine Mantelhälfte gelähmt worden war, entweder beide 
Flossen mit ihrer Haut, oder abpräparierte und auf einen Korkrahmen aufgespannte Stücke von der gelähmten und normalen Rückenhaut nebeneinander als Vergleichsobjelite benützte. Sepiahaut, die einige Zeit in nicht durchlüftetem Seewasser liegt, bleicht, wenn sie vorher dunkel war, langsam aus. Bringt man sie dann wieder an die Luft, so tritt, insbesondere auf der gelähmten Seite, nach einiger Zeit Dunkelung ein, indem sich die Chromatophoren allmählich expandieren. Auf der normalen Seite pflegt die Expansion, wenn man stärkeres Eintrocknen verhütet, lange nicht so bedeutend zu sein als auf der gelähmten ${ }^{1}$ ). Lässt man zu solcher Haut, die in einer Glasschale liegt, Kohlensäure zuströmen, so bemerkt man zunächst eine zunehmende Dunkelung und nachher Auftreten von Wolkenwandern - wie gesagt, fast ausschliesslich auf der Haut der gelähmten Seite. Dabei nimmt jetzt die Haut im ganzen einen immer blässeren, gelblichen Farbenton an und bleicht bei sehr reichlicher Kohlensäurezufubr nach längerer Zeit schliesslich ganz aus, indem auch das Wolkenwandern immer spärlicher wird und endlich erliseht. Zutritt frischer Luft und Verjagung der Kohlensäure ruft dann von neuem Wolkenwandern hervor, wobei nunmehr die vorher bleiche Haut wieder im ganzen dunkler wird. Der oben erwähnte gelbliche Ton rührt davon her, dass zuerst die dunkelvioletten Chromatophoren sich retrahieren, während die gelben noch expandiert sind. Dabei sieht man nun in diesem Stadium, in welchem die gelben Chromatophoren nicht mehr wie sonst.von den dunkeivioletten verdeckt sind, dass auch die gelben Chromatophoren für sich allein dasselbe Wolkenwandern zeigen wie die violetten.

Das Wolkenwandern besteht, wie schon bemerkt wurde, in einem ganz kontinuierlichen Fortschreiten der Erregung. Die Richtung, in welcher der dunkle Fleck, die "Wolke", wandert, ist ganz unregelmässig. Gelegentlich biegt sie um und kehrt auf einem Umwege wieder auf eine frühere Stelle zurück. Manche Gebiete werden be-

1) Durch welchen Reiz die Chromatophorenerregung verursacht wird, ist :schwer zu sagen. Man denkt natürlich zuerst an das langsame Eintrocknen der Haut und die infolgedessen allmählich zunehmende Konzentration des sie befeuchtenden Seewassers. Doch tritt die Erregung auch auf, wenn man die Haut fleissig mit so reichlichen Mengen von Seewasser befeuchtet, dass der Überschuss abläuft. Eine wichtige Vorbedingung für das Zustandekommen der Chromatophorenerregung ist der ungehinderte Zutritt des Luftsauerstoffs zur Haut. Weiteres darüber folgt in der nächsten Abhandlung. 
vorzugt, an manchen erlischt sie. Manchmal kehren die Wolken längere Zeit hindurch immer wieder auf derselben Strecke in derselben Richtung wieder. Zumeist treten die Wolken, wenn einmal die Bedingungen für sie gegeben sind, ganz von selbst, ohne weiteres Zutun, auf. Doch kann man durch Reizung einer Hautstelle diese zum Ausgangspunkt einer Erregungswelle machen. Setzt man durch mechanische Reizung einen Dauereffekt (vgl. dazu die nächste Abhandlung!), so können von dieser Stelle aus mehrere Wolken hintereinander ausgehen. Auch eine Chromatophorenexpansion, welche indirekt durch Nervenreizung hervorgerufen wurde, kann zum Ausgangsort einer Wolke werden. Nie beobachtet wird beim Wolkenwandern das in der nächsten Abhandlung genauer beschriebene, für die Erregung der Nervenbündel des Grundplexus charakteristische Hinüberspringen der Erregung über unerregte Gebiete. Ferner ist beachtenswert, dass das Wolkenwandern der in etwas verschiedener Höhe liegenden violetten und gelben Chromatophoren gesondert für sich abläuft. Die Erregung pflanzt sich demnach beim Wolkenwandern nur in den allerletzten Endgliedern des motorischen Apparates der Chromatophoren fort, also entweder in einem kontinuierlichen Nervenendnetz, das in so unmittelbarem Zusammenhange mit den Muskelfasern stehen müsste, dass jede Erregung desselben auch mit einer Muskelerregung verknüpft ist, oder in hypothetischen Anastomosen zwischen den Muskelfasern selbst. Die letztere Annahme hat Steinach zur Erklärung des Wolkenjagens herangezogen. Er sagt (1. c. S. 19): „Da die Fortpflanzung solcher Wellen und das Übergreifen der Bräunung auch auf ungereizte Hautpartien, wie sich unten ergibt, von nervösen Vorgängen nicht abhängig ist, so sind wir berechtigt, diese Irradiationen einerseits auf die erwähnten Anastomosen der Radiärfaserenden mit denen benachbarter Chromatophoren, andererseits auf die vorgefundenen Übergänge der Radiärfasern in die Hautmuskelbündel zurückzuführen" ${ }^{1}$ ). Stein ach schliesst deswegen so, weil er mittels seiner 1. c. gleich darauf beschriebenen "Degenerationsmethode" alle nervösen Apparate ausgeschaltet zu haben glaubt, und trotzdem noch Pulsationen und Wellenspiel der Chromatophoren fortbestand. Ich kann mich dieser Folgerung von Steinach nicht anschliessen. Steinach's

1) Ich habe die Befunde, auf welche Steinach hier hinweist, schon in der histologischen Abhandlung zitiert. 
"Degenerationsmethode" bestand darin, dass er abgeschnittene Fangarme von Oktopus oder Eledone im Dunkeln in öfter gewechseltem Seewasser so lange aufbewahrte, bis sowohl jeder Reizerfolg bei Tetanisierung des nervösen Achsenstranges der Arme als auch jeder Reflex auf Hautreizung hin verschwunden war. Ausgeschaltet ist dann zweifellos der Einfluss der nervösen Zentralorgane und der gröberen, im Innern des Armes verlaufenden Nervenstämme. Dagegen. lässt sich nicht ohne weiteres sagen, ob nicht die letzten Nervenverzwejgungen in der Haut noch funktionsfähig geblieben sind. Zunächst beobachtet man, dass in den Nerven der Cephalopoden beim Absterben ein zunehmend stärkeres Dekrement auftritt. Selbst wenn man also voraussetzt, dass alle Nervenstrecken mit gleicher Geschwindigkeit absterben, so wird einmal der Fall eintreten, dass zwar bei Reizung des Achsenstranges die Erregung nicht mehr bis zu den Chromatophoren vordringt, wohl aber noch bei Reizung der Nerven in der Peripherie. (Das Absterben schreitet scheinbar vom Zentrum gegen die Peripherie zu fort.) Sodann stehen ja dio Endausbreitungen der Chromatophorennerven in der Haut unter ganz. anderen Bedingungen als die Nerven der tieferen Teile. Wie aus. den Beobachtungen, die in der folgenden Abhandlung mitgeteilt werden sollen, hervorgeht, ist die Haut der Cephalopoden für Gase, insbesondere auch für Sauerstoff, sehr gut durchlässig, und es werden demnach die Hautgewebe, wenn die Haut an der Luft liegt, auch nach dem Tode noch mit 0 versorgt. Diese Sauerstoffversorgung ist zwar vielleicht nicht so ausgiebig wie am lebenden Tiere mit erhaltenem Kreislauf ${ }^{1}$ ), aber sie reicht doch hin, um die Hautgewebe noch lange nach dem Tode funktionsfähig zu erhalten. Für die Nervenplexus der Chromatophoren von Sepia lässt sich direkt zeigen, dass sie noch reizbar bleiben, wenn Reizung der grösseren Nervenstämme, welche zur Haut hinziehen, keinen Erfolg mehr gibt. Dieser Beweis ist oben S. 392 schon geführt worden, und ich brauche daher hier bloss auf jene Stelle zu verweisen.

Aus den angeführten Gründen kann ich demnach nicht finden, dass durch das Steinach'sche Verfahren die Mitbeteiligung der

1) Die Hautatmung spielt äbrigens gewiss bei diesen Tieren auch im Leben eine grosse Rolle, denn es ist sicher sehr zweckmässig, wenn ausser den eigentlichen Atemorgancn auch die ganze Hantoberfläche an der Saucrstoffaufnahme: beteiligt ist. 
Nervengeflechte in der Haut am Wolkenwandern mit Sicherheit allisgeschlossen ist, und man musste sich daher danach umschauen, ob sich nicht andere Kriterien zur Entscheidung zwischen den vorliegenden beiden Möglichkeiten auffinden liessen. Da ist nun die Tatsache von höchstem Interesse, dass alle in der folgenden Abhandlung aufgeführten nervenlähmenden Gifte, wenn sie auf die Haut aufgetragen werden (Chloralhydrat) oder. nach subkutaner Injektion (Kokain, Atropin) ungefähr zur selbenZeit, wenn sie die indirekte Reizbarkeit der Chromatophoren a ufheben, auch das Wolkenwandern auf ihrer Einwirkungsstelle zum Erlöschen bringen. Die Erregungswellen, welche von unvergifteten Stellen ankommen, pflanzen sich auf die vergiftete Stelle nicht mehr fort. Daraus kann man nach den Ausführungen der nächsten $\mathrm{Ab}$ handlung schliessen, dass die Erregung beim Wolkenwandern eniweder innerhalb der nervösen Substanz fortschreitet, oder wenn intramuskulär, dann wenjgstens in einer der nervösen ausserordentlich ähnlichen Substanz. Dies ist nun freilich keine Entscheidung unserer Frage, sondern sie führt vielmehr unsere hier aufgeworfene Spezialfrage in jenen ganzen Komplex strittiger Punkte hinüber, der bezüglich des Verhaltens der Nervenendigungen zu den Muskelfasern in cler mesenchymatösen Muskulatur uberhaupt besteht. Aber ich bin sogar der Meinung, dass selbst eine klare Entscheidung dieser Hauptstreitfrage noch keine völlige Aufk]ärung des hier in Rede stehenden Punktes bietet. Für diese ist es nämlich eigentlich ganz gleichgültig, ob man ein kontinuierliches Nervenendnetz oder Anastomosen zwischen den Muskelzellen annimmt. Die Hauptfrage ist doch die, wieso es denn kommt, dass diese Verbindungen, seien sie nun nervöser oder "muskulärer" Natur, nachdem sie unter normalen Verhältnissen die Erregung nicht oder mit enormem Dekrement leiten, unter den abnormen Verbältnissen, welche zum Wolkenwandern führen, auf einmal eine so gute Leitfähigkeit bekommen, dass von einem Dekrement so gut wie gar. keine Rede ist! Nach den Ausführungen der histologischen Abhandlung gibt es doch zum mindesten ganz sicher ein Anastomosennetz zwischen den Endverzweigungen eines Neurons unter sich. Wie kommt es denn, so wird man verwundert fragen, dass nicht jedesmal, wenn man an irgendeiner kleinen Stelle, z. B. durch mechanische Reizung, eine Erregung setzt, diese nicht zum mindesten über das ganze Innervationsgebiet des 
betreffenden Neurons hinwegläuft? Oder gar, wenn ein kontinuierliches Nervenendnetz vorbanden ist, warum erzeugt nicht jede lokale Erregung auch Wolkenwandern? Und im anderen Falle, was bcfähigt die supponierten Anastomosen zwischen den einzelnen Muskelfasern unter gewissen Umständen zu einem so guten Leitungsvermögen? Hier also, sieht man, steckt gerade der springende Punkt.

Ich bin nun durch die mikroskopische Beobachtung pulsierender Chromatophoren von Loligo vulgaris, an welehen die Nerven zum grössten Teile schon mit Methylenblau gefärbt waren, auf eine Erklärung geführt worden, welche ich schon in meinem Vortrage auf dem internationalen Physiologenkongress zu Brüssel ${ }^{1}$ ) erwähnt habe, und welche ich auch jetzt, nach reiflichem Durchdenken, noch nicht für endgültig abgetan halte. Wir wissen zunächst, dass bei Sepia das Wolkenwandern auf weite Strecken, scheinbar ganz ohne Dekrement, nur an seit längerer Zeit gelähmter Haut auftritt, an welcher, wie in der folgenden Abhandlung gezeigt werden soll, die mechanische Reizbarkeit der Nervenfasern des Grundplexus ausserordentlich gesteigert ist. Nun ziehen ja die Radiärmuskeln bei ihrer Kontraktion nicht bloss am Chromatophorenkörper, sondern sie üben. einen Zug auch nach der anderen Seite auf die Nervenfasern aus, welche vom Grundplexus her $\mathrm{zu}$ ihnen verlaufen. Man muss es. selbst unter dem Mikroskope gesehen haben, wie deutlich sich dieser Zug in einer Zerrung, der Nervengeflechte äussert ${ }^{2}$ ), um es völlig begreiflich zu finden, dass dadurch eine Erregung der Nervenfasern. gesetzt wird. Man müsste sich geradezu wundern, wenn dies bei der so hochgradigen mechanischen Reizbarkeit auf der gelähmten. Seite nicht der Fall wäre. Damit ist aber gerade die Hauptfrage, die nach der Fortpflanzung der Erregung ohne Dekrement, gelöst: Wenn jede sich kontrahierende Muskelfaser überhaupt imstande ist, durch den mechanischen Reiz der Kontraktion selbst die benachbarten Nervengeflechte zu erregen, so wird die Erregung so weit wandern, als die Reizbarkeit der angrenzenden Nervenfasern genügend hoch ist. Sobald diese so niedrig wird, dass der Dehnungsreiz unter der

1) Résumé des Vortrags im Archivio di fisiol. vol. 2 p. 116. 1904 und in den Arch. internat. de physiol. vol. 2 p. 101. 1905.

2) Nebenbei bemerkt, gehört diese Erscheinung zu den reizendsten Naturerscheinungen, die ich je zu beobachten Gelegenheit hatte. Ich kann nur jedem raten, sich, wenn die Möglichkeit vorliegt, selbst davon zu überzeugen. Die Unter-suchungsmethode ist ja einfach genug (vgl. die histologische Abhandlung!). 
Schwelle bleibt, muss die Erregung ganz ausbleiben, die Erregungswelle muss rapid abbrechen. Bei dieser Auffassung ist es ganz gleichgültig, welche speziellen Vorstellungen man sich über die Endigung der Nerven an der glatten Muskulatur macht, ja im Grunde genommen wäre es sogar einerlei, ob man sich den Dehnungsreiz am Nerven oder an den Muskeln angreifend denkt. Freilich wäre die ganze Erklärung hinfällig, wenn sie nur auf die speziellen Verbältnisse bei Sepia zugeschnitten wäre. Denn das Wolkenwandern tritt ja auch an der Haut von Oktopus und Eledone unter Bedingungen auf, zu denen eine vorherige länger dauernde Lähmung nicht gehört. Aber schon Steinach hat auseinandergesetzt (I. c. S. 22), dass unter diesen Bedingungen auch bei Oktopus und Eledone durch Dehnung der Haut (stranmes Anspannen) das Wellenspiel hervorgerufen wird. Wenn man also auch in diesem Falle mit den v. Frey'schen Reizhaaren vielleicht keine so hohe Reizbarkeit der Chromatophorennerven nachweisen kann, wie an der gelähmten Sepiahaut, so ist jedenfalls als erwiesen zu betrachten, dass auch dort der Dehnungsreiz überhaupt wirksam ist, demnach die oben gegebene Erklärung wahrscheinlich ebenfalls zutrifft.

Nun mag freilich in allen Fällen noch etwas anderes hinzukommen. An der Sepiahaut sieht man das Wolkenwandern immer dann auftreten, wenn eine vorhandene scheinbar spontane (d. h. durch noch unbekannte Reize hervorgerufene) Erregung der Chromatophoren durch irgendeinen Eingriff eben unterdrückt wird, oder wenn nach diesem Eingriff die Erregung eben wieder zurückkehrt. An der Luft liegende Sepiahaut wird dunkel, bei Koblensäureeinwirkung bleicht sie unter Wolkenwandern aus. Ebenso bleicht sie mit anfänglichem Wolkenwandern aus, wenn man durch ein aufgelegtes Deckgläschen die O-Zufuhr von der Luft aufhebt; Wolkenwandern sieht man gelegentlich bei der ersten Einwirkung nervenlähmender Gifte, so nach Atropinvergiftung, und am regelmässigsten nach subkutaner Injektion von mit Salzsäure neutralisiertem Physostigmin $^{1}$ ); Wolken wandern tritt endlich auf, wenn man die durch Kohlensäure gebleichte Haut wieder an die Luft bringt und sie nun allmählich wieder dunkelt. Kurz, es scheint, dass man alle diese Fälle in den Satz

1) Subkutane Injektion von neutralisiertem Physostigmin in etwa $3 / 4 \%$ iger Lösung in Seewasser ist das einzige mir. bekannte Mittel, Wolkenwandern auch auf der nicht gelähmten Sepiahaut hervorzurufen. 
zusammenfassen kann: Wolkenwandern tritt jedesmal auf, 'wenn eine schwache Reizung vorhanden ist, welche unter den gegebenen Erregbarkeitsverhältnissen eben nicht mehr über die Schwelle treten kann. Kommt in diesem Falle ein anderer, wenn auch an sich schwacher Reiz hinzu, so bricht die Erregung aus. Dass dieser Komplementreiz in der Dehnung der Chromatophorennerven besteht, scheint mir nach dem oben Erwähnten doch wohl möglich zu sein.

Ganz ohne Bedenken ist diese Auffassung freilich nicht. Dass man beim Wolkenwandern auf dér gelähmten Haut kein solches Hinüberspringen über unerregte Stellen beobachten kann, wie bei mechanischer Reizung durch Druck von aussen, das ist freilich kaum verwunderlich, wenn man bedenkt, dass von der Zerrung durch die Radiärmuskeln wohl nicht der eigentliche Grundplexus, sondern vielmehr die Übergangsfasern und die Fasern des Endplexus betroffen werden, deren mechanische Reizbarkeit gewiss ebenfalls gesteigert ist. Eher ist es bedenklich, dass man zwar selten, aber doch mitunter auch auf normaler Haut ganz rudimentäres kurzes Wolkenwandern sehen kann, ja es sugar durch.subkutane Injektion von neutralisiertem Physostigmin hervorrufen kann, trotzdem ich dabei bisher jene so hochgradige mechanische Reizbarkeit wie auf der gelähmten Seite noch nicht gefunden habe. Aber vielleicht genügt, wie bei Oktopus und Eledone, schon ein geringerer Grad derselben, dessen Nachweis mir bloss noch nicht gelungen ist. Am wichtigsten scheint mir aber der Umstand, dass die Wanderungsgeschwindigkeit der Wolken in hohem Maasse von der Temperatur abbängt. Bringt man ein Stück gelähmter Sepiahaut aus Seewasser von etwa $15^{\circ} \mathrm{C}$. in Seewasser, das auf ungefähr $30^{\circ} \mathrm{C}$. erwärmt ist, so tritt spontan oder auf Reizung hin Wolkenwandern auf, aber mit einer ganz enorm grossen Wanderungsgeschwindigkeit, welche die Geschwindigkeit bei Zimmertemperatur, wie man schon ohne Messung sagen kann, um ein Mehrfaches übertrifft. Nun läge hier entschieden die Annahme am nächsten, dass die Erregungsleitung beim Wolkenwandern durch ein Nerven- oder Muskelnetz erfolgte, und dass die Geschwindigkeit der Erregungsleitung in diesem Netz von der Temperatur abhinge. Will man aber die Meinung aufrecht erhalten, dass der Dehnungsreiz den Vermittler des Fortwanderns der Wolke macht, dann muss man zu etwas komplizierteren Annahmen greifen: Man kann nämlich darauf hinweisen, dass ja durch den Dehnungsreiz zunächst Nervenfasern in Erregung versetzt werden, dass die 
Erregung in diesen erst bis zu den nächsten Radiärfasern weiter geleitet werden, und dann auf den Muskel übertragen werden muss. Wenn nun die Erregungsleitung in diesen Nervenfasern durch Erwärmung beschleunigt wird, wenn dabei überdies auch das mechanische Latenzstadium des Muskels verkürzt wird und der Kontraktionsakt rascher abläuft ${ }^{1}$ ), somit auch die Dehnung rascher erfolgt, so kann dies alles auch ein viel rascheres Wandern der Erregungswelle bewirken. Man könnte vielleicht sogar darüber streiten, welche der beiden Annahmen zu einer ausgiebigeren Verkürzung der Leitungszeit führt.

Man sieht aus diesen Überlegungen, dass die „Dehnungshypothese" durch solche Zweifel und Bedenken jedenfalls noch nicht streng ausgeschlossen ist. Das wäre sie nur dann, wenn sich nachweisen liesse, dass der Kontraktionsvorgang als solcher für das $\mathrm{Zu}$ standekommen des Wolkenwanderns nicht nötig ist. Es würde sich also darum handeln, ein Mittel zu finden, das Kontraktionsvermögen an einer Stelle auszuschalten, ohne die Reizbarkeit zu stark herabzusetzen und dann zuzusehen, ob die Erregungswelle trotzdem noch über die sich nicht kontrahierende Stelle hinwegwandert oder nicht. Diese Forderung klingt vielleicht übertrieben, scheint mir aber nicht ganz unausführbar zu sein, und ich werde bei nächster Gelegenheit versuchen, $o b$ sich solche Versuchsbedingungen nicht doch berstellen lassen. Sollte sich herausstellen, dass wirklich der mechanische Reiz der Dehnung zur Fortpflanzung der Erregung nicht notwendig ist, dann wäre allerdings das Wolkenwandern von allerhöchstem Interesse. Denn dann würde der bemerkenswerte Fall vorliegen, dass man experimentell eine kontinuierliche Erregungsleitung hervorrufen könnte in einem Mesenchymmuskel, in welchem die Fähigkeit dazu sonst gewissermaassen latent ist und unter normalen Umständen gar nicht zum Vorschein kommt. Ich brauche uicht weiter auszuführen, welche Wichtigkeit dies für das Studium. der Innervation der glatten Muskulatur bei Wirbeltieren haben würde.

Schliesslich möchte ich noch hinzufügen, dass sich der Hautmuskel in jeder Beziehung der Chromatophorenmuskulatur analog verhält: Man kann durch Reizung eines Nervenbündels isolierte Innervationsgebiete darstellen,' die sich nur wegen der passiven Zerrung der Umgebung nicht so scharf abgrenzen lassen wie bei den

1) Letzteres ist zweifellos der Fall. 
Chromatophoren. Man beobachtet ferner an längere Zeit gelähmter Haut unter denselben Bedingungen wie an der Chromatophorenmuskulatur wandernde Erregungswellen, die oft zu sehr heftiger Verschiebung der Haut führen. Bezüglich der Ursache des Wolkenwanderns kann man in beiden Fällen auf die Zerrung der Umgebung rekurrieren; kurz, wir finden am Hautmuskel in allem wesentlichen das gleiche Verhalten wie an der Chromatophorenmuskulatur. Da aber durch die Beobachtungen am Hautmuskel etwas wesentlich Neues zum Vorigen nicht hinzugefügt werden kann, so kann ich mich hier auf diesen kurzen Hinweis beschränken.

\section{Schlussbemerkungen.}

Das Resultat, zu welchem unsere Experimente schliesslich gefübrt haben, dass sich unter normalen Umständen für jeden zur Chromatophoren-, Flossen- und Mantelmuskulatur der Cephalopoden hinziehenden Nerven gesonderte Innervationsgebiete nachweisen lassen, über welche der Einfluss des betreffenden Nerven nicht hinausreicht, stimmt überein mit den Beobachtungen über die Innervation der glatten und der ihr verwandten Muskulatur der Wirbeltiere ${ }^{1}$ ). So äussert sich bezüglich der Innervation der Irismuskeln und der glatten Muskeln an den Haaren Langle ${ }^{2}$ ) folgendermaassen: „In the iris and in the skin it is certain, that nervous impulses entering at any one point affect a limited and local area." Nachdem er einige diesbezügliche Experimente angeführt hat, fährt er fort: ${ }_{n}$ Consequently there cannot be either in the skin or in the iris any considerable spreading out of impulses by means of a nervous network. If any such spreading out of impulses occurs it can only occur in the small area to which direct fibres run, and in which it is superfluous to assume it. The experimental evidence is, I think, in favour of isolated conduction in the postganglionic branches" $\ldots$...

Als besonders bemerkenswert kann ich hinzufügen die Be-

1) Ganz ebenso liegen offenbar die Verhältnisse auch bei anderen wirbellosen Tieren, so z. B. nach den Untersuchungen von V. Henri (Comptes rend. de la Soc. de Biol. t. 55 p. 1194. 1904) an den Längsmuskeln der Holothurien. Bei Stichopus regalis bewirkte lokalisierte mechanische, thermische, chemische und elektrische Reizung stets nur eine lokale Kontraktion, die sich nie über die ganze Länge des Muskels ausbreitete. Vgl. ferner Biedermann, Pflüger's Arch. Bd. 102 S. 487 ff. und Bd. 107 S. 35 ff.

2) On the question of commissural fibres between nerve cells having the same function etc. Journ. of Physiol. vol. 31 p. 257. 1904.

E. Pflĩ ger, Archiv für Physiologie. Bd. 118. 
obachtungen am Herzmuskel, bemerkenswert schon deshalb, weil hier auch die histologische Paralleluntersuchung sehr vollständig durchgeführt ist. Diese hatte das Resultat ergeben ${ }^{1}$ ), dass es entschieden am nächsten läge, „ein ganz kontinuierliches, die ganze Muskulatur vom Sinus bis zum Ventrikel und Bulbus durchziehendes Nervennetz anzunehmen". Nun besteht, was immer man sonst noch für Annahmen machen mag, jedenfalls ein Teil dieser Nervengeflechte aus den Endigungen der regulatorischen Herznerven. Für die Hemmungsnerven habe ich dies ja direkt histologisch und physiologisch nachweisen können ${ }^{2}$ ). Die Endausbreitung der Erregung in den regulatorischen Herznerven ist nun genau wieder eine so lokalisierte, wie die Innervation der sonstigen "mesenchymatösen Muskulatur" (Brüder Hertwig). So habe ich für die Hemmungsfasern nachgewiesen ${ }^{3}$ ), dass nach der Durchschneidung der Scheidewandnerven des Froschherzens Vagusreizung trotz bochgradiger Abschwächung der Vorhofskontraktionen gar keine Wirkung mehr auf den Ventrikel gibt, obwohl, wie gesagt, histologisch das Nervennetz in der Verbindungsmuskulatur zwischen Vorhof und Ventrikel genau ebenso schön und anscheinend kontinuierlich entwickelt ist wie in der Vorhofs- und Ventrikelmuskulatur selbst. Dieselbe lokalisierte Innervation hat jüngst in ganz ähnlicher Weise auch H. E. Hering ${ }^{4}$ ) beim Säugetierherzen für den Accelerans nachgewiesen. Es ist also auch im Herzen ausgeschlossen, dass sich die hemmende oder fördernde Nervenwirkung in einem intramuskulären Endnetz, selbst wenn es existieren sollte, fortpflanzt, vielmehr bestehen auch hier für die postganglionären Nervenfasern isolierte Innervationsgebiete. Ich brauche nur auf meine Ausführungen über die wahrscheinliche Verknüpfung der Hemmungsfasern im Herzen zu einer funktionellen Einheit hinzuweisen ${ }^{5}$ ), um die Wichtigkeit dieser Erkenntnis klarzulegen.

1) Das intrakardiale Nervensystem des Frosches. His' Arch. 1902 S. 103.

2) Die neurogene und myogene Theorie der Herztätigkeit etc. Schmidt's Jahrb. d. Medizin Bd. 281 s. 117 ff. 1904.

3) Beiträge zur Lehre von der Herzinnervation. PfI üger's Arch. Bd. 72 S. 437 und 459.1898.

4) Über die unmittelbare Wirkung des Accelerans und Vagus anf automatisch schlagende Abschnitte des Säugetierherzens. Pflüger's Arch. Bd. 108 S. 289 ff. 1905 .

5) Schmidt's Jahrb. Bd. 281 S. 221.1904. 


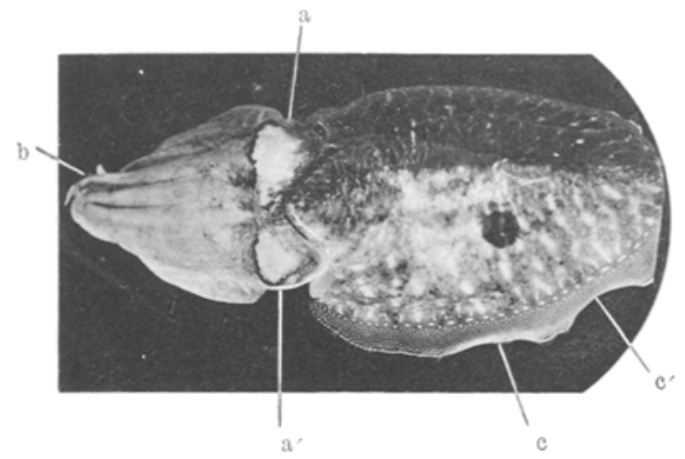

Fig. 1.

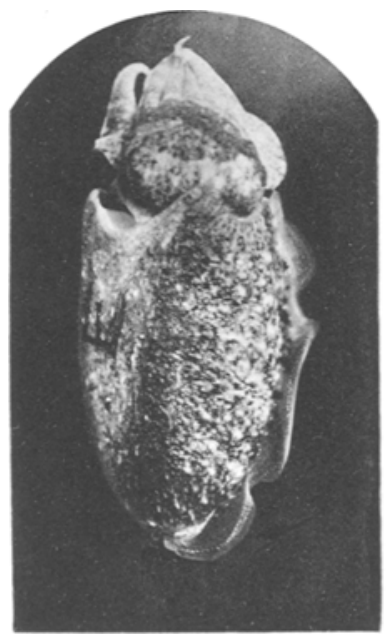

Fig. 2.

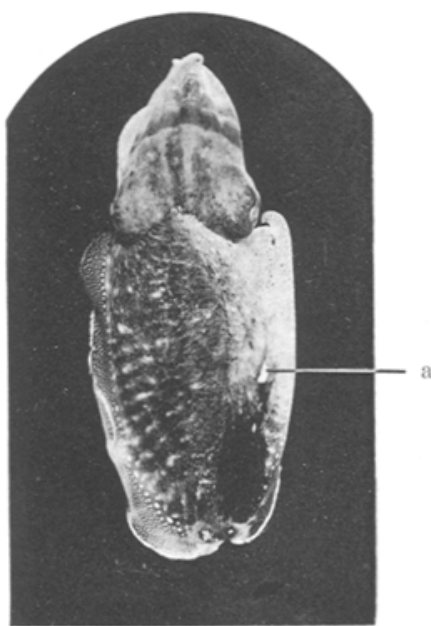

Fig. 3. 